\title{
Lactate signalling regulates fungal $\beta$-glucan masking and immune evasion
}

Elizabeth R. Ballou, Gabriela M. Avelar, Delma S. Childers, Joanna Mackie, Judith M. Bain, Jeanette Wagener, Stavroula L. Kastora, Mirela D. Panea, Sarah E. Hardison, Louise A. Walker, Lars P. Erwig, Carol A. Munro, Neil A.R. Gow, Gordon D. Brown, Donna M. MacCallum, and Alistair J.P. Brown*

\author{
Aberdeen Fungal Group, MRC Centre for Medical Mycology, \\ School of Medicine, Medical Sciences and Nutrition, \\ Institute of Medical Sciences, University of Aberdeen, \\ Foresterhill, Aberdeen, AB25 2ZD, UK.
}

* Corresponding author:

Telephone: (+44) 1224-555883

Fax: (+44)1224-555844

Email: al.brown@abdn.ac.uk

Running head: Fungal immune evasion

Key words: Candida albicans; lactate sensing; signal transduction; PAMP masking; immune evasion; fungal pathogenicity; RNA sequencing. 


\section{Summary Paragraph}

As they proliferate, fungi expose antigens at their cell surface that are potent stimulators of the innate immune response, and yet the commensal fungus Candida albicans is able to colonize immuno-competent individuals. We show that $C$. albicans may evade immune detection by presenting a moving immunological target. We report that the exposure of $\beta$-glucan, a key Pathogen Associated Molecular Pattern (PAMP) located at the cell surface of $C$. albicans and other pathogenic Candida species, is modulated in response to changes in carbon source. Exposure to lactate induces $\beta$-glucan masking in $C$. albicans via a signaling pathway that has recruited an evolutionarily conserved receptor (Gpr1) and transcriptional factor (Crz1) from other well-characterized pathways. In response to lactate, these regulators control the expression of cell wall related genes that contribute to $\beta$-glucan masking. This represents the first description of active PAMP masking by a Candida species, a process that reduces the visibility of the fungus to the immune system.

Vertebrates recognize microbial pathogens via immunological synapses between host Pattern Recognition Receptors (PRRs) and the corresponding Pathogen Associated Molecular Patterns (PAMPs) ${ }^{1}$. Many PAMPs lie at the cell surface, the first point of contact with the host. The fungal cell wall is a matrix of $\alpha$ - and $\beta$-mannans, phosphomannans, $\beta$-glucan, and chitin, each of which exerts immuno-modulatory effects $^{1}$. $\beta-1,3-$ glucan, an essential component of most fungal cell walls, is a ligand for the C-type lectin receptor Dectin- $1^{1}$. $\quad \beta$-glucan recognition by Dectin-1 triggers phagocytosis and killing by neutrophils and macrophages ${ }^{2}$. Some fungi have evolved strategies to prevent this interaction. For example, Cryptococcus neoformans expresses an anti-inflammatory polysaccharide capsule that masks $\beta$-glucan ${ }^{3}$. The RodA hydrophobin masks $\beta$-glucans at the Aspergillus fumigatus spore surface ${ }^{4}$. We show that pathogenic Candida species actively mask surface $\beta$-glucan in response to the host metabolite lactate, thereby attenuating the recognition of Candida albicans by host phagocytes. 
C. albicans possesses an elastic cell wall in which $\beta$-glucan is generally buried below an outer fibrillar layer of mannan, within an inner matrix of cross-linked glucan and chitin $^{5}$. However, $\beta$-glucan becomes exposed at bud growth sites, during yeast-hypha morphogenesis, exposure to antifungal drugs, and within biofilms ${ }^{6-9}$. Moreover, $\beta$-glucan exposure increases during systemic infection ${ }^{7}$, in a remodeling process partly mediated by host cell antifungal activity ${ }^{10}$. Indeed, the $C$. albicans cell wall undergoes significant remodeling in response to host-derived inputs including changes in carbon source, oxygen levels and iron availability ${ }^{11,12}$, or after in vitro exposure to antifungal drugs ${ }^{7}$.

Specific signaling pathways mediate $C$. albicans cell wall remodeling in response to genetic perturbation or antifungal drug treatments ${ }^{7}$. These include the Mkc1 cell integrity pathway, the Hog1 stress response pathway, the Cek1 pathway, and $\mathrm{Ca}^{++}$calcineurin (Cna1) signaling ${ }^{13-15}$. However, the signaling pathways that drive cell wall remodeling in response to nutritional signals remain obscure.

Changes in carbon source lead to alterations in cell wall structure, affect immune surveillance and enhance the virulence of $C$. albicans ${ }^{12,16}$. The carbon sources available to support fungal growth and colonization differ between host niches. Some contain glucose (e.g. blood), some lack glucose (e.g. the colon), while others contain significant concentrations of carboxylic acids such as lactate (e.g. the vagina and colon) ${ }^{17,18}$. Here we define the mechanisms by which carbon source affects $\beta$-glucan exposure and immune surveillance. We show that $C$. albicans exploits conserved signaling elements to actively mask this major PAMP in response to the physiologically relevant carboxylic acid L-lactate. Furthermore, we show that this phenomenon is conserved in other pathogenic Candida species.

\section{Lactate triggers $\beta$-glucan masking in pathogenic Candida species}

The architecture of the $C$. albicans cell wall changes significantly with carbon source: cells grown on lactate possess thinner, less elastic cell walls compared to glucose-grown cells ${ }^{12,19}$. Although the proportion of $\beta$-glucan relative to mannan and chitin remains similar ${ }^{12}$, we reasoned that $\beta$-glucan organization and exposure at the C. albicans cell surface might be affected by carbon source. To test this, cells were stained with Fc-Dectin-1, and median fluorescence intensity (MFI) was quantified by flow cytometry. Glucose-grown cells displayed a biphasic distribution of $\beta$-glucan exposure (Figures 1ai). The low exposure population generally contained small cells, whereas 
larger cells demonstrated higher levels of $\beta$-glucan exposure, consistent with previously described mothers with bud scars ${ }^{7,9}$. In contrast, lactate-grown cells displayed a monophasic population of smaller, proliferating mother and daughter cells (Figures 1ai). Glucose-grown cells reproducibly displayed higher levels of $\beta$-glucan exposure than lactate-grown cells. Significantly, cells grown on a mixture of glucose plus lactate, which were similar in size to glucose-grown cells, exhibited reduced levels of $\beta$-glucan exposure, equivalent to lactate-grown cells (Figures $1 \mathrm{~b}$, Supplementary Figure 1a). Therefore, lactate triggers $\beta$-glucan masking even in the presence of glucose. (We defined $\beta$-glucan masking as a $\geq 50 \%$ reduction in MFI.) These changes in $\beta$-glucan exposure are carbon source-dependent, not pH-dependent (Supplementary Figure 1b). $\beta$-glucan masking is retained over long periods (Supplementary Figure 1c), but is reversible (Supplementary Figure 1d): $\beta$-glucan exposure increased within 1 hour ( $<1$ generation) of transferring lactate-grown cells to glucose, whilst $\beta$-glucan masking occurred within 6 hours of adding lactate to glucose-grown cells and was retained throughout 72 hours growth on lactate ( $\sim 51$ generations).

This lactate-induced $\beta$-glucan masking phenotype was observed in almost all C. albicans clinical isolates examined (Supplementary Figure 2). (The molecular basis for the single exception, Clade 4 strain S20175.016, is discussed below). These blood,

oral, vaginal, nail, and animal isolates were from eight epidemiological clades ${ }^{20}$. Other pathogenic yeasts in the CTG clade including C. albicans, C. dubliniensis, C. Iusitaniae, C. parapsilosis and $C$. orthopsilosis displayed lactate-induced $\beta$-glucan masking (Figure 1aii-iv). Candida glabrata is more closely related to $S$. cerevisiae than to the other Candida species, yet masking was observed for $C$. glabrata but not S. cerevisiae (Figure lav,vi). Therefore, many pathogenic Candida species display lactate-dependent masking, suggesting that this phenotype might promote immune evasion, and hence a pathogenic lifestyle.

\section{Growth of $C$. albicans on lactate reduces phagocytic recruitment}

Lactate-grown C. albicans cells stimulate less IL-17 production by human peripheral blood mononuclear cells (PBMCs), and are phagocytosed less efficiently by J774.1 macrophages than glucose-grown cells ${ }^{16}$. We recapitulated these observations with murine bone marrow-derived macrophages and also demonstrated that lactate attenuates neutrophil recruitment during infection in vivo (Figures 1c,d). Lactate-grown 
cells recruited fewer neutrophils to the intraperitoneal space than glucose-grown cells $(p=0.0022)$ (Figure 1d). No difference was observed in the total number of $C D 45^{+}$ immune cells collected $(p=0.94)$, but the recruitment of $\left(C D 11 b^{+}\right.$Ly6 $\left.C^{\text {high }}\right)$ inflammatory monocytes was increased (Figure S3a). Furthermore, lactate-grown cells stimulated less TNF $\alpha(p=0.0005)$ and less MIP1 $\alpha(p<0.0001)$ from human M1-activated monocytederived macrophages than glucose-grown cells (Figures 1e,f, Supplementary Figure $3 b, c)$. Therefore, lactate-grown $C$. albicans cells stimulate altered immune responses compared to glucose-grown cells. This is consistent with our observation that lactategrown cells are more virulent than glucose-grown cells in a murine model of systemic candidiasis $^{12}$.

\section{$\beta$-glucan masking does not depend on lactate metabolism}

Lactate exists as two isomers, D- and L-lactate. C. albicans does not metabolize D-lactate, but assimilates L-lactate, the form generated by the host. Gastrointestinal microbiota secrete D- and L-lactate. The above work used a racemic mixture of D/Llactate (1:1), and therefore we tested each enantiomer separately. $\beta$-glucan masking was triggered by L-lactate, but not by its D-isomer (Figure 2a). Furthermore, $\beta$-glucan masking was activated by spent culture medium from macrophages (L-Lactate) or the gut bacterium Lactobacillus reuteri, (D- and L-Lactate) (Figure $2 b$ ). Therefore, $\beta$-glucan masking is triggered by the lactate isomer of physiological relevance to both host niches and the gut microbiota.

We tested whether lactate metabolism is essential for $\beta$-glucan masking. In C. albicans lactate assimilation depends on uptake via the Jen $1 / 2$ transporters ${ }^{21}$ and the glyoxylate cycle enzyme isocitrate lyase $(\mathrm{Icl} 1)^{17}$. Lactate-induced $\beta$-glucan masking persisted in icl1 $\Delta$ single and jen $1 \Delta$ jen2 $\Delta$ double mutants (Supplementary Figure 4). Therefore, $\beta$-glucan masking is not dependent on lactate metabolism.

\section{Gpr1 is a lactate sensor in C. albicans}

Lactate sensors have been described in mammals and fish. For example, lactate stimulates lipolysis in mammalian adipocytes via the G-protein coupled receptor GPR81, and key lactate binding residues have been defined ${ }^{22}$. We identified Gpr1 as the closest C. albicans orthologue of human GPR81. In C. albicans, Gpr1 and its Ga protein Gpa2 
activate CAMP signaling to promote yeast-hypha morphogenesis and virulence ${ }^{23}$. In S. cerevisiae, the Gpr1 ligand is glucose, and its glucose binding site has been defined experimentally $^{24}$. The ligand binding regions from ScGpr1 and CaGpr1 diverge at key residues (Supplementary Figure 5), consistent with a change in ligand specificity. Indeed, methionine, rather than glucose, has been proposed as the CaGpr1 ligand ${ }^{23}$.

Given the structural relatedness between L-lactate and L-methionine (Supplementary Figure 5), we tested whether Gpr1 mediates lactate-induced $\beta$-glucan masking. Masking was lost in the gpr1 $\Delta$ gpa2 $\Delta$ double mutant (Figure 2c), significantly attenuated in gpr $1 \Delta$ cells and partially attenuated in gpa2 $\Delta$ cells relative to their CAI4 parent (Figure 2c, Supplementary Figure 6a,c), indicating that this phenotype is dependent on Gpr1 and partially on its G-alpha protein Gpa2. As described above, C. albicans S20175.016 did not display lactate-induced $\beta$-glucan masking (Supplementary Figure 2). Sequencing of both GPR1 alleles from this strain revealed non-synonymous SNPS relative to GPR1 from SC5314. Transforming GPR1 from SC5314 into S20175.016 was sufficient to restore lactate-induced $\beta$-glucan masking (Figure 2c), confirming that Gpr1 is required for this phenotype.

We examined the impact of lactate on the localization of Gpr1-GFP in C. albicans. Gpr1-GFP localizes to the cell surface in C. albicans grown on minimal medium containing glucose ${ }^{23}$. In our hands, Gpr1-GFP localized to the cell surface and vesicles under these conditions (Figure 2di), suggesting dynamic cycling of this receptor. Following lactate addition, Gpr1-GFP was internalized (Figure 2div-vi). Methionine also triggers Gpr1-GFP internalization ${ }^{23}$. We recapitulated this response, but only at nonphysiological concentrations $(100 \mathrm{mM})$, and methionine did not activate $\beta$-glucan masking (Figure 2diii,d). Pyruvate promoted partial masking, which was lost in the gpr1s gpa2s background (Figure 2f, Supplementary Figure 6b). Neither proline nor acetate triggered $\beta$-glucan masking, or promoted Gpr1-GFP internalization (Figure 2e,f). In contrast, lactate consistently stimulated robust Gpr1-GFP internalization and $\beta$-glucan masking at low concentrations (2mM, 10mM: Figure 2div,v) in a Gpr1-Gpa2-dependent manner (Supplementary Figure 6b). These data highlight the specificity of Gpr1dependent lactate-induced masking. 


\section{Lactate-induced $\beta$-glucan masking does not depend on cAMP signaling}

C. albicans Gpr1 and Gpa2 act upstream of the cAMP pathway to activate yeasthypha morphogenesis ${ }^{23}$. Therefore, we examined lactate-induced $\beta$-glucan masking in C. albicans cyr $1 \Delta$ cells lacking adenylyl cyclase that are defective in cAMP signaling and display cell wall defects. These cells exhibited abnormally high $\beta$-glucan exposure (Supplementary Figure 7a). However, lactate still induced $\beta$-glucan masking (Supplementary Figure 7a). Likewise, $\beta$-glucan masking persisted in pde2 $\Delta$ cells lacking the inhibitory cyclic phosphodiesterase (Supplementary Figure 7a). Furthermore, exogenous cAMP did not influence $\beta$-glucan masking (Supplementary Figure 7a). We conclude that, despite the involvement of Gpr1, lactate-induced $\beta$-glucan masking is not mediated by cAMP signaling.

These data suggest that Gpr1 plays dual roles in morphogenetic regulation ${ }^{23}$ and lactate-induced $\beta$-glucan masking (Figure 2). In other systems G-protein coupled receptor specificity is mediated by ligand-specific interaction with G-alpha subunits ${ }^{25}$. We therefore investigated the role of the second G-alpha subunit, Cag1. A cag1 $\Delta$ strain exhibited constitutive $\beta$-glucan masking (Supplementary Figure 6a,c), suggesting that Cag1 plays an inhibitory role in this process. Therefore, Cag1 and Gpa2 may modulate the specificity of Gpr1 signaling to the $\beta$-glucan masking and cAMP pathways via mechanisms that remain to be defined.

We further tested whether key morphogenetic signaling pathways mediate lactateinduced $\beta$-glucan masking. Cells lacking Efg1 and Cph1, which regulate hyphal development and are targets of CAMP and MAPK signaling, respectively, displayed normal lactate-induced $\beta$-glucan masking (Supplementary Figure 7b). Furthermore, inactivation of Cek1, an ERK-family protein kinase that regulates Cph1 and influences cell wall structure and $\beta$-glucan ${ }^{15}$, did not perturb masking (Supplementary Figure 7c).

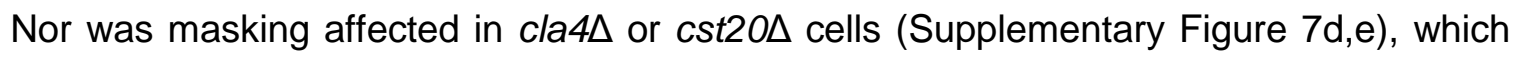
lack Ste20p-like kinases for Cek1-Cph1 signaling ${ }^{26}$. Therefore, lactate-induced $\beta$-glucan masking is not dependent on morphogenetic signaling via the MAPK or cAMP pathways.

\section{A Crz1-dependent pathway triggers lactate-induced $\beta$-glucan masking}

To identify regulators of lactate-induced $\beta$-glucan masking, we examined transcription factors known to influence cell wall organization. This revealed the 
involvement of $\mathrm{Crz1}$, which regulates membrane integrity, antifungal drug tolerance, and cell wall remodeling ${ }^{13,27}$. Lactate-induced $\beta$-glucan masking was blocked in C. albicans $\operatorname{crz1} \Delta$ cells, and this defect was suppressed by $C R Z 1$ reintegration (Figure $3 a$ ).

In C. albicans, Crz1 is activated by the calcineurin pathway in response to calcium and cell wall stress. Under these conditions, calcineurin signaling is blocked by deletion of CNA1 (encoding the catalytic subunit of calcineurin) or CNB1 (encoding the regulatory subunit), or by pharmacological inhibition of calcineurin by $\mathrm{FK} 506^{28}$. However, lactateinduced $\beta$-glucan masking was not perturbed in cna1s or cnb1s cells or in wild-type cells treated with FK506 (Figure 3a), suggesting that lactate-dependent Crz1 activation is not mediated via calcineurin.

Calcineurin controls Crz1 localization in response to calcium ${ }^{28}$. In C. albicans, $\mathrm{Ca}^{2+}$ triggers calcineurin-mediated dephosphorylation of $\mathrm{Crz1}$, leading to its nuclear accumulation $^{28}$. We recapitulated this observation (Figure $3 \mathrm{~b}$ ) and observed that lactate also triggers nuclear accumulation of GFP-Crz1 (Figure 3b). Significantly, the nuclear accumulation of GFP-Crz1 was reduced in gpr1 1 gpa2 $\Delta$ cells in response to lactate, but not in response to calcium (Figure 3b). In contrast, loss of CNB1 prevented GFP-Crz1 nuclear accumulation in response to calcium, but not in response to lactate (Figure 3b). We conclude that Crz1 is activated by alternative signaling pathways depending on the nature of the input signal. Whilst Crz1 is activated via calcineurin in response to calcium $^{28}$, the response to lactate is mediated via Gpr1. This signaling is not dependent on the protein kinase $\mathrm{C}$ cell integrity pathway (Supplementary Figure 8).

\section{Lactate-dependent targets of Crz1 include cell wall functions}

We performed genome-wide transcriptional profiling to identify genes that might contribute to lactate-induced $\beta$-glucan masking in $C$. albicans and investigate the impact of the Crz1 transcription factor. Using RNA sequencing, we compared glucose alone and glucose plus lactate cells, which grow at similar rates (Supplementary Table 1). The 157 lactate-regulated genes we identified were significantly enriched $\left(p<10^{-5}\right)$ in functions related to the plasma membrane, cell surface, and cell periphery, consistent with the changes in cell wall $\beta$-glucan exposure (Supplementary Table 2). Furthermore, the regulation of two-thirds of these 157 genes was compromised by $C R Z 1$ inactivation, suggesting that they are either direct or indirect targets of Crz1 (Supplementary Table 1). ACE2, a transcription factor regulating cell wall integrity, protein mannosylation and 
chitin synthesis ${ }^{29}$, was induced in $\operatorname{crz} 1 \Delta$ cells exposed to lactate. Also, we identified overlaps between Ace2 targets identified by ChIPSeq ${ }^{30}$ and the genes highlighted by our RNA sequencing (Supplementary Table 3). Therefore, the influence of ACE2 on lactateinduced $\beta$-glucan masking was tested. We examined ace $2 \Delta$ cells by confocal and fluorescence microscopy because their aggregation, due to a severe cytokinesis defect $^{29}$, precludes flow cytometry. The ace $2 \Delta$ cells displayed high levels of $\beta$-glucan exposure in the presence of lactate (Figure $4 a, b)$, indicating a block in masking. This suggests the involvement of a Crz1-Ace2 network in lactate-induced $\beta$-glucan masking (Figure 5, Supplementary Table 3).

We tested whether lactate-regulated cell wall genes influence lactate-induced $\beta$ glucan masking, reasoning that the outer mannan layer might contribute to masking ${ }^{31,32}$ (Figure $4 a, b)$. Mnn14 $\Delta$ cells did exhibit a partial masking defect $(26.2 \%$ decrease in MFI; we define masking as a $>50 \%$ decrease) (Figure $4 a$ ). However, mnn22 $\Delta$ and exg2 $\Delta$ cells retained the masking phenotype (Figure $4 a, b$ ), and although the levels of $\beta$ glucan exposure were elevated for cht2 $\Delta$ and pga26 $\Delta$, lactate still triggered masking (Figure $4 a, b)$. Also, our microscopic analyses of wild-type, gpr1 1 gpa2 $\Delta$ and $\operatorname{crz} 1 \Delta$ cells did not reveal a simple inverse relationship between mannan distribution and $\beta$-glucan exposure at the cell surface (Figure 2g,h, 3c). Therefore major changes in mannan distribution do not appear to account for lactate-induced $\beta$-glucan masking. We tested whether key cell wall crosslinking enzymes are required for this phenotype by examining a triple crh11 $\Delta$ crh $12 \Delta$ utr $2 \Delta$ mutant that lacks three cell wall glycosidases. These cells displayed a gross increase in glucan exposure, but retained some degree of $\beta$-glucan masking (Supplementary Figure 9), suggesting that Crh11, Crh12 and Utr2 are not essential for masking.

\section{Discussion}

We show that L-lactate, generated by host cells or bacteria in the host's microbiota, actively triggers masking of a major PAMP at the fungal cell surface (Figure 1). This lactate-induced $\beta$-glucan masking phenotype, which is observed in a range of pathogenic Candida species (Figure 1), is triggered by physiologically relevant 
concentrations of lactate and is not activated by other host-derived metabolites such as acetate, methionine, or proline (Figure 2). This PAMP masking correlates with reduced uptake of $C$. albicans cells by macrophages in vitro, decreased stimulation of TNFa and MIP1a by human macrophages, and diminished recruitment of neutrophils in vivo (Figure 1).

Carbon source-mediated immune evasion is likely to be significant in the context of host colonization because $C$. albicans cells adapt rapidly to the mixtures of carbon sources offered by host niches (Figure 2, Supplementary Figure 1$)^{17}$. Lactate levels can reach 2-4 mM in blood and vaginal secretions, and can comprise approximately $2 \%$ of all carbon metabolites generated by the gut microbiota ${ }^{33}$. Glucose is essentially absent from the colon, present at 3-5 $\mathrm{mM}$ in the blood, and at concentrations of up to $20 \mathrm{mM}$ in vaginal secretions ${ }^{11,18,34}$. Although changes in additional PAMPs may contribute to the effects of carbon source, the importance of fungal $\beta$-glucan exposure and Dectin-1mediated fungal recognition is widely recognized ${ }^{35-38}$. Taken together, the data indicate that changes in PAMP exposure occur as the fungus adapts to local nutrients within host niches, and this affects the visibility of the colonising fungus to host immune defences. This idea is consistent with the observations that $\beta$-glucan exposure changes during the progression of systemic candidiasis ${ }^{7,9}$, that $C$. albicans adaptation affects immune recognition by Dectin-1 during systemic candidiasis ${ }^{20}$, and that changes in carbon source alter the virulence of $C$. albicans ${ }^{12}$.

How does lactate trigger $\beta$-glucan masking? We show that lactate triggers a signaling pathway that has co-opted the Gpr1 G-protein coupled receptor and the Crz1 transcription factor from the CAMP and calcineurin signaling pathways, respectively (Figures 2 and 3). Maiden et al. showed that the Gpr1-Gpa2 module triggers filamentation in response to amino acid signals in a cAMP independent fashion ${ }^{23}$. We hypothesize that a parallel pathway involving Gpr1, and possibly Gpa2, mediates lactate signaling to Crz1. This pathway does not require calcineurin, which is reminiscent of undefined calcineurin-independent modes of $\mathrm{Crz1}$ regulation in other fungi ${ }^{39,40}$. Crz1 then appears to regulate the expression of targets including ACE2 (Table S1), the inactivation of which affects lactate-induced $\beta$-glucan masking (Figure 4). Deletion of ACE2 also leads to $\beta$-glucan exposure at the $S$. cerevisiae cell surface ${ }^{6}$. In $C$. albicans, Crz1 regulates additional cell wall genes that may collectively mediate $\beta$-glucan masking (Figures 4,5, Supplementary Table 1). However, individual cell wall mutations did not block this phenotype. Taken together these observations support the view that PAMP 
masking is a polygenic trait that is influenced by cumulative changes in the quantity, crosslinking and organization of multiple cell wall macromolecules ${ }^{6}$, and that the expression of these traits is modulated by lactate-Gpr1-Crz1 signaling (Figure 5).

How is $\beta$-glucan masked at the cell surface? $\beta$-glucan is generally buried below the outer fibrillar layer of the $C$. albicans cell wall, and is therefore masked by mannans. Mutations that compromise the mannan outer layer or glucan cross-linking cause $\beta$ glucan exposure at the cell surface (Figure 4). Mannan attachment to the inner glucanchitin layer is dependent on cell wall remodeling enzymes whose expression is affected by growth on different carbon sources ${ }^{41}$. Therefore, mannans play a general role in $\beta$ glucan masking and may contribute to lactate-induced masking. However, mutants with defects in individual cell wall genes are still capable of lactate-induced masking, suggesting that $\beta$-glucan masking is dependent on overall changes in cell wall architecture.

In conclusion, this study reveals that $C$. albicans actively masks a major PAMP in response to host-derived carbon sources, essentially turning $C$. albicans into a moving target for the immune system in response to host-specific signals. This type of immune evasion is likely significant for disease progression in the host during periods of anaerobic metabolism (when lactic acid production is high), in the gastrointestinal tract (where anaerobic bacteria generate high lactate concentrations), and in patients with diabetes, short-bowel syndrome or bacterial infection (when serum lactate concentrations become elevated). This precedent, where C. albicans exploits host cues to modulate its immune visibility, may apply to other fungal pathogens (Figure 1$)^{42,43}$.

\section{Methods}

Strains and culture conditions. Strains used in this study are listed in Table S4. In all cases, WT indicates the prototrophic parent of a given mutant strain, with the exception of SN152, for which auxotrophy has been demonstrated to have no impact on fungal virulence $^{44}$. C. albicans was routinely cultured on YPD (1\% yeast extract, $2 \%$ bactopeptone, $2 \%$ glucose, $2 \%$ bacto-agar) agar plates stored at room temperature. For glucose and lactate exposure, cells were incubated overnight at $30^{\circ} \mathrm{C}, 200 \mathrm{rpm}$ in $5 \mathrm{~mL}$ 
YNB without amino acids (Sigma Y1250) prepared according to the manufacturer's instructions plus $2 \%$ glucose and/or $2 \%$ D/L-lactate (Sigma, L1375) as indicated. All auxotrophic strains were grown in media with appropriate supplements.

BMDM phagocytosis assay. All experiments were conducted by approval of the College of Life Sciences and Medicine Ethics Review Board, University of Aberdeen, and they all adhered to local and institutional policy requirements. C57BL/6 wild-type mice were bred and housed in the University of Aberdeen animal facility. Female mice aged 21 weeks were used to generate bone marrow derived macrophages (BMDM) by flushing femurs and tibia with RPMI 1640 GlutaMAX (Thermo Fisher Scientific, Waltham, MA, USA) supplemented with $10 \%(\mathrm{v} / \mathrm{v}$ ) heat-inactivated foetal calf serum (FCS; SigmaAldrich, Dorset, UK), $200 \mathrm{U} \mathrm{ml}^{-1}$ penicillin/streptomycin (Sigma), $10 \mathrm{mM} \mathrm{HEPES} \mathrm{(Sigma).}$ Bone marrow cells were cultured for 7 days at $37^{\circ} \mathrm{C}$ with $5 \% \mathrm{CO}_{2}$ in supplemented RPMI plus $15 \%$ (v/v) Lee's conditioned medium. Following cell counts, $1.2 \times 10^{5}$ BMDM in $300 \mu$ l supplemented RPMI medium were seeded into 8-well imaging slides (ibidi, Munich, Germany) to adhere overnight. Phagocytosis assays were performed by combining 3:1 thimerosal-fixed yeast:adhered macrophages as described previously ${ }^{45}$. Immediately prior to imaging, wells were replenished with fresh $\mathrm{CO}_{2}$-independent medium (Thermo Fisher Scientific) supplemented with FCS and glutamine (Sigma). Conditions were maintained at $37^{\circ} \mathrm{C}$ with $5 \% \mathrm{CO}_{2}$ during $4 \mathrm{~h}$ image acquisition at 1 minute intervals using an UltraVIEW ${ }^{\circledR}$ VoX spinning disk microscope (Nikon, Surrey, UK) with Volocity ${ }^{\circledR}$ software (Improvision, PerkinElmer, Coventry, UK). Triplicate movies were analysed for percent phagocytosis at 4 hours post inoculation for each condition with 4 independent experimental replicates conducted (a total of 200-300 macrophages combined with approx. 600-800 yeast per condition). Data were analysed by MannWhitney U test using GraphPad Prism 7 (GraphPad Software Inc., La Jolla, CA, USA). Variance within the groups was not statistically different ( $F$ test to compare variances, $\mathrm{p}=0.1253)$.

Monocyte isolation, purification and macrophage differentiation. Blood from four healthy volunteers was collect according to the local guidelines and regulations, approved by the College Ethics Review Board of the University of Aberdeen and following informed consent of the donors (CERB/2012/11/676). Peripheral blood 
mononuclear cells (PBMCs) were isolated by Ficoll-Paque PLUS (GE Healthcare) density centrifugation according to the manufactures instructions. Highly pure unlabelled monocytes were obtained from PBMCs by depletion of non-monocytes using a magnetically activated cell sorting (MACS) system together with a human pan-monocyte isolation kit (Miltenyi Biotec). Purified monocytes were washed twice with PBS and cells were suspended in RPMI 1640 (Dutch modification) supplemented with 10\% heatinactivated FBS, $2 \mathrm{mM}$ L-glutamine, $1 \mathrm{mM}$ sodium pyruvate, 1\% MEM non-essential amino acids (NEAA (100x), Gibco), $100 \mathrm{U} / \mathrm{ml}$ penicillin, $100 \mathrm{mg} / \mathrm{ml}$ streptomycin and $50 \mathrm{ng} / \mathrm{ml}$ recombinant hGM-CSF (Gibco). Cells were seeded into cell culture dishes at a density of $1 \times 10^{6}$ cells $/ \mathrm{cm}^{2}$, incubated at $37^{\circ} \mathrm{C}$ in a humidified atmosphere containing $5 \%$ $\mathrm{CO}_{2}$. On day $310 \mathrm{ml}$ fresh medium containing recombinant cytokines was added to the cultures. After 5 days cells were collected, seeded into 24-well plates at a density of $1 \times 10^{5}$ cells/well and left overnight to adhere. Macrophages were fully activated with 50 ng/ml IFN-g and $100 \mathrm{ng} / \mathrm{ml}$ LPS 24 hours prior to co-culture with formaldehyde fixed $C$. albicans yeast.

On the day of co-culture, macrophages were washed with PBS and fresh medium added. Then, C. albicans was pre-grown in YNB Glucose, Glucose + Lactate, or Lactate for 4 hours as described, fixed with 4\% methanol-free formaldehyde (Thermo), and washed 3x with sterile 1xPBS (Sigma), was added to each well at an MOI of 5:1 macrophages. For each donor, PBS only wells were also included. At times 0, 6, and 24 hr post infection, $100 \mathrm{uL}$ of supernatant was collected from each well and stored at -20 . Cytokines and chemokines were quantified using the Luminex® Screening kit (R\&D) and read on a BioPlex 200 System (Bio-Rad) according the manufacturer's recommendations. Statistical analyses were performed using Graph Pad Prism (v 7), and significance was determined using the Friedman test for matched non-parametric data. Bars represent $95 \% \mathrm{Cl}$.

Neutrophil recruitment assay. BALB/c female mice ( $n=6 / g r o u p, ~ 8-12$ weeks old, Harlan, UK) were injected intraperitoneally with $10^{7} \mathrm{C}$. albicans (SC5314) pre-grown in either glucose or lactate and then resuspended in $100 \mu \mathrm{L}$ sterile saline. Group size was determined from previous experiments as the minimum number of mice needed to detect statistical significance $(p<0.05)$ with $90 \%$ power. Mice were randomly assigned to groups by an investigator not involved in the analysis and the fungal inocula were 
randomly allocated to groups. Mice were house in Individually Ventilated Cages (IVCs) and were provided with food and water ad libitum. Inocula were delivered in an unblinded fashion. After $4 \mathrm{~h}$, mice were sacrifice using the schedule 1 method cervical dislocation. Immune infiltrates were collected by peritoneal lavage ${ }^{46}$. Cells were labeled to distinguish neutrophils (CD45 $\left.{ }^{+}, \mathrm{CD} 11 \mathrm{~b}^{+}, \mathrm{F} 4 / 80^{-}, \mathrm{Ly}^{+} \mathrm{G}^{+}\right)$and macrophages (CD45 ${ }^{+}$,

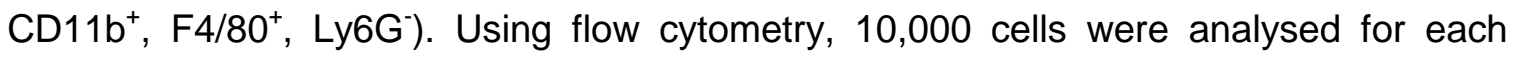
mouse. Cells were analysed on a BD Fortessa flow cytometer, with automatic compensation protocols. Data represent percent neutrophils. Statistical analyses were performed using Graph Pad Prism ( $v$ 7), and significance was determined using MannWhitney $\mathrm{U}$ test. Bars represent $95 \% \mathrm{Cl}$. Variance within the groups was not statistically different ( $F$ test to compare variances, $p=0.1633$ ). All animal experiments were performed under UK Home Office project license PPL 60/4135 granted to DMM in accordance with Home Office ethical guidelines. These experiments were performed by DMM, ERB, and SEH.

Quantification of $\beta$-glucan exposure in cell populations. Yeasts were grown in YNB $+2 \%$ glucose, $+2 \%$ glucose and $2 \%$ lactate, or $2 \%$ lactate overnight and inoculated into fresh medium for 4 hours. Cells were stained for $\beta$-glucan exposure using Fc-Dectin- ${ }^{47}$. Cells were counted by haemocytometer and $2.5 \times 10^{6}$ cells were washed with cold FACS buffer (1xPBS, 1\% FCS, 0.5 mM EDTA). Cells were resuspended in $100 \mu \mathrm{L}$ FACS buffer $+5 \mathrm{ng} / \mu \mathrm{l}$ Dectin-Fc and incubated in the dark on ice for $1 \mathrm{~h}$. Cells were washed (5000 rpm, 3 min) twice in $100 \mu \mathrm{L}$ FACS Buffer and resuspended in $100 \mu \mathrm{L}$ FACS buffer plus 1:200 Gt F(ab')2 anti-Human IgG(gamma) conjugated to Alexafluor 488 (Invitrogen, cat. No. H10120) or Novex TRITC (Abcam, cat. No. A18822), for GFP-expressing cells. Cells were incubated in the dark on ice for $1 \mathrm{~h}$ and washed as above. Cells were fixed in $4 \%$ Formaldehyde (methanol- free), diluted in FACS Buffer, and stored on ice in the dark until analysis using either a FACS Calibur flow cytometer or BD Fortessa flow cytometer. For each experiment, 10,000 events were acquired for each sample. Fresh glucosegrown C. albicans SC5314 cells were used as a positive control for each run. As a secondary-only control, aliquots from all cells to be analysed were pooled, diluted to 2.5 $x 10^{6}$ cells, and treated as above except that no Dectin-Fc was added. Median Fluorescence Intensity (MFI) is reported for each sample as determined using FlowJo v 10 software. This measure was selected as the most appropriate for the bimodal data 
observed for cells grown in glucose. Masking was defined as more than $50 \%$ decrease in MFI based on 6 independent biological replicates of glucose vs. glucose-lactate grown cells. Plots are representative of at least two biological replicates for strains with no masking defect and at least three biological replicates for strains with an observed masking defect.

Spent culture media. J774.1 macrophages (ECACC, HPA, Salisbury, UK) were resuspended from liquid nitrogen stocks and grown to confluency in RPMI-1640 + Lglutamine $+10 \%$ FCS-HI $+100 \mathrm{U} / \mathrm{mL}$ Penicillin $+100 \mu \mathrm{g} / \mathrm{ml}$ Streptomycin. Cells were split and incubated for 3 days in fresh medium. The supernatant was collected and passed through a $0.45 \mu \mathrm{m}$ filter. C. albicans SC5314 was pre-grown in YNB plus 2\% glucose, washed with 1XPBS, and incubated in fresh medium or spent, filtered supernatant for 6 hours. Cells were stained for $\beta$-glucan as described above.

L. reuteri isolated from pig gut was a generous gift from Dr Karen Scott (Rowett Institute of Nutrition and Health, University of Aberdeen). L. reuteri was incubated overnight in M2 medium (Sigma) at $37^{\circ} \mathrm{C}$, ambient air, $200 \mathrm{rpm}$. Cells were spun down and the supernatant was collected and passed through a $0.45 \mu \mathrm{m}$ filter. C. albicans SC5314 was pre-grown in YNB plus 2\% glucose, washed with 1XPBS, and incubated in fresh M2 or spent, filtered supernatant for 6 hours. Cells were stained for $\beta$-glucan as described above and 10,000 cells were analyzed by Flow Cytometry; $n=2$.

Plasmid construction. GFP-tagged proteins were generated by cloning the relevant ORF into CIp-NAT-GFP, which uses the CIp-NAT backbone ${ }^{48}$ with the GFP ORF flanked by Age1 and Kas1 restriction sites to facilitate in-frame cloning under control of the ACT1 promoter. The GPR1 ORF was inserted at the Age1 restriction site to generate GPR1-GFP. The CRZ1 ORF was inserted in-frame at the Kas1 restriction site to generate GFP-CRZ1.

RNA extraction and analysis. Cells were incubated overnight in $5 \mathrm{ml}$ volumes in the indicated conditions and transferred to fresh medium in $50 \mathrm{~mL}$ culture flasks for $4 \mathrm{~h}$ (30 ${ }^{\circ} \mathrm{C}, 200 \mathrm{rpm}$ ) to an $\mathrm{OD}_{600}=0.6$. RNA was obtained via Qiazol/chloroform extraction (QIAgen) according to the manufacturer's instructions and assessed for quality by 
NanoDrop 1000 (Thermo Scientific). RNA was DNAse treated (TURBO DNAse, Ambion) according to the manufacturer's instructions and assessed for quality by Agilent Bioanalyser $(\mathrm{RIN}>8)$. Samples were processed through the lon Torrent Proton sequencer (CGEBM University of Aberdeen Genomics facility). Raw fastq files were successively processed in the following order through Fastqc (v. 10.1), Trimgalore ( $v$. 3.1), Samtools (v. 1.19), STAR aligner (v. 2.4) and Htseq (v. 5.4). Genome alignment was conducted against the C_albicans_SC5314_version_A21-s02-m09-r08 chromosomes file provided by the Candida Genome Database (http://www.candidagenome.org/) ${ }^{49}$. Aligned data were quality controlled via the Partek Genomics Suite ${ }^{\circledR}$ software, version 6.6, Copyright 2015, as per manufacturer's instructions. Biological replicates grouped by genotype and growth medium, and exhibited similar variance within each condition. Gene expression analysis was performed using Partek ${ }^{\circledR}$ Genomics Suite ${ }^{\circledR}$ software using a $\log _{2}$ data transformation as the Partek® recommended default. GO term analysis was performed in parallel through the Candida Genome Database GO Term Finder and the Cytoscape v. 3 Clue GO plugin $^{50}$. Network construction was performed with Cytoscape V.3 freeware ${ }^{51}$. Statistical comparison among GO term enrichment percentages was performed with Graph Pad Prism (v. 6) using Student's T-test for two-tailed data. Data represent three independent biological replicates for each condition.

Data availability. The RNA sequencing dataset is available at $\mathrm{EBI}$ (http://www.ebi.ac.uk/arrayexpress/) under accession number E-MTAB-4579. Other data that support the findings of this study are available from the corresponding author upon reasonable request.

\section{Acknowledgements}

All correspondence and requests for materials should be addressed to Alistair Brown. We apologize to colleagues whose work we have been unable to cite because of space constraints. We thank Frank Odds for access to his collection of clinical isolates. We are grateful to Raif Yuecel and Linda Duncan in the lain Fraser Cytometry Centre (Aberdeen University), and Ms Lucinda Wight and $\mathrm{Mr}$ Kevin MacKenzie in the Microscopy and Histology Core Facility (Aberdeen University) for their expert help with the cytometry and microscopy experiments. We are also grateful for the assistance of 
staff at the University of Aberdeen Medical Research Facility. We also thank $\mathrm{Mr}$ Brennan Martin in the Centre for Genome Enabled Biology and Medicine (Aberdeen University) for help with the RNA sequencing.

\section{Author contributions}

E.R.B., D.S.C., G.M.A. and A.J.P.B. planned the experiments. E.R.B., G.M.A., D.S.C., J.M., J.M.B., J.W., M.D.P. and D.M.M. performed the experiments. S.L.K. and E.R.B performed bioinformatic analyses. S.E.H, L.A.W., L.P.E., C.A.M, N.A.R.G. and G.D.B. provided materials and valuable insight during this study. E.R.B. and A.J.P.B. drafted the manuscript with contributions from J.M., G.M.A., D.S.C., J.M.B., C.A.M, N.A.R.G., G.D.B. and D.M.M..

\section{Funding:}

AJPB: This work was supported by the European Research Council (STRIFE, ERC2009-AdG-249793), The UK Medical Research Council (MR/M026663/1), the UK Biotechnology and Biological Research Council (BB/K017365/1), the Wellcome Trust (080088; 097377). ERB: This work was supported by the UK Biotechnology and Biological Research Council (BB/M014525/1). GMA: Supported by the CNPq-Brazil (Science without Borders fellowship 202976/2014-9). GDB: Wellcome Trust (102705). CAM: This work was supported by the UK Medical Research Council (G0400284). DMM: This work was supported by UK National Centre for the Replacement, Refinement and Reduction of Animals in Research (NC/K000306/1). NARG/JW: Wellcome Trust (086827, 075470,101873) and Wellcome Trust Strategic Award in Medical Mycology and Fungal Immunology (097377). ALL: This work was supported by the MRC Centre for Medical Mycology and the University of Aberdeen (MR/N006364/1). 


\section{References}

1 Brown, G. D. \& Gordon, S. Immune recognition. A new receptor for beta-glucans. Nature 413, 36-37, doi:10.1038/35092620 (2001).

2 Hardison, S. E. \& Brown, G. D. C-type lectin receptors orchestrate antifungal immunity. Nature immunology 13, 817-822, doi:10.1038/ni.2369 (2012).

3 O'Meara, T. R. \& Alspaugh, J. A. The Cryptococcus neoformans capsule: a sword and a shield. Clin Microbiol Rev 25, 387-408, doi:10.1128/CMR.00001-12 (2012).

4 Carrion, S. d. J. et al. The RodA hydrophobin on Aspergillus fumigatus spores masks dectin-1- and dectin-2-dependent responses and enhances fungal survival in vivo. Journal of Immunology (Baltimore, Md : 1950), 191, 2581-2588, doi:10.4049/jimmunol.1300748 (2013).

5 Gow, N. A. R. \& Hube, B. Importance of the Candida albicans cell wall during commensalism and infection. Current Opinion in Microbiology 15, 1-7, doi:10.1016/j.mib.2012.04.005 (2012).

6 Wheeler, R. T. \& Fink, G. R. A drug-sensitive genetic network masks fungi from the immune system. PLoS Pathogens 2, e35, doi:10.1371/journal.ppat.0020035 (2006).

7 Wheeler, R. T., Kombe, D., Agarwala, S. D. \& Fink, G. R. Dynamic, morphotypespecific Candida albicans beta-glucan exposure during infection and drug treatment. PLoS Pathogens 4, e1000227, doi:10.1371/journal.ppat.1000227 (2008).

8 Nett, J. E., Sanchez, H., Cain, M. T. \& Andes, D. R. Genetic basis of Candida biofilm resistance due to drug-sequestering matrix glucan. The Journal of Infectious Diseases 202, 171-175, doi:10.1086/651200 (2010).

9 Gantner, B. N., Simmons, R. M. \& Underhill, D. M. Dectin-1 mediates macrophage recognition of Candida albicans yeast but not filaments. Embo J 24, 1277-1286, doi:10.1038/sj.emboj.7600594 (2005).

10 Hopke, A. et al. Neutrophil Attack Triggers Extracellular Trap-Dependent Candida Cell Wall Remodeling and Altered Immune Recognition. PLoS Pathog 12, e1005644, doi:10.1371/journal.ppat.1005644 (2016).

11 Sosinska, G. J. et al. Hypoxic conditions and iron restriction affect the cell-wall proteome of Candida albicans grown under vagina-simulative conditions. Microbiology (Reading, England) 154, 510-520, doi:10.1099/mic.0.2007/012617-0 (2008).

12 Ene, I. V. et al. Host carbon sources modulate cell wall architecture, drug resistance and virulence in a fungal pathogen. Cellular Microbiology 14, 13191335, doi:10.1111/j.1462-5822.2012.01813.x (2012).

13 Munro, C. A. et al. The PKC, HOG and Ca2+ signalling pathways co-ordinately regulate chitin synthesis in Candida albicans. Mol Microbiol 63, 1399-1413, doi:10.1111/j.1365-2958.2007.05588.x (2007).

14 Heilmann, C. J. et al. Surface stress induces a conserved cell wall stress response in the pathogenic fungus Candida albicans. Eukaryotic Cell 12, 254-264, doi:10.1128/EC.00278-12 (2013). 
15 Galán-Díez, M. et al. Candida albicans beta-glucan exposure is controlled by the fungal CEK1-mediated mitogen-activated protein kinase pathway that modulates immune responses triggered through dectin-1. Infect Immun 78, 1426-1436, doi:10.1128/IAI.00989-09 (2010).

16 Ene, I. V., Cheng, S.-C., Netea, M. G. \& Brown, A. J. P. Growth of Candida albicans Cells on the Physiologically Relevant Carbon Source Lactate Affects Their Recognition and Phagocytosis by Immune Cells. Infect Immun 81, 238-248, doi:10.1128/IAI.01092-12 (2013).

17 Barelle, C. J. et al. Niche-specific regulation of central metabolic pathways in a fungal pathogen. . Cellular Microbiology 8, 961-971, doi:10.1111/j.14625822.2005.00676.x (2006).

18 Owen, D. H. \& Katz, D. F. A vaginal fluid simulant. Contraception 59, 91-95 (1999).

19 Ene, I. V. et al. Cell Wall Remodeling Enzymes Modulate Fungal Cell Wall Elasticity and Osmotic Stress Resistance. mBio 6, e00986, doi:10.1128/mBio.00986-15 (2015).

20 Marakalala, M. J. et al. Differential adaptation of Candida albicans in vivo modulates immune recognition by dectin-1. PLoS Pathogens 9, e1003315, doi:10.1371/journal.ppat.1003315 (2013).

21 Vieira, N. et al. Functional specialization and differential regulation of short-chain carboxylic acid transporters in the pathogen Candida albicans. Molecular Microbiology 75, 1337-1354, doi:10.1111/j.1365-2958.2009.07003.x (2010).

22 Kuei, C. et al. Study of GPR81, the Lactate Receptor, from Distant Species Identifies Residues and Motifs Critical for GPR81 Functions. . Molecular Pharmacology 80, 848-858, doi:10.1124/mol.111.074500 (2011).

23 Maidan, M. M. et al. The G protein-coupled receptor Gpr1 and the Galpha protein Gpa2 act through the cAMP-protein kinase A pathway to induce morphogenesis in Candida albicans. Mol Biol Cell 16, 1971-1986, doi:10.1091/mbc.E04-09-0780 (2005).

24 Lemaire, K., Van de Velde, S., Van Dijck, P. \& Thevelein, J. M. Glucose and sucrose act as agonist and mannose as antagonist ligands of the $G$ proteincoupled receptor Gpr1 in the yeast Saccharomyces cerevisiae. Molecular Cell 16, 293-299, doi:10.1016/j.molcel.2004.10.004 (2004).

25 Johnston, C. A. \& Siderovski, D. P. Receptor-Mediated Activation of Heterotrimeric G-Proteins: Current Structural Insights. . Molecular Pharmacology 72, 219-230., doi: http://doi.org/10.1124/mol.107.034348 (2007).

26 Leberer, E. et al. Virulence and hyphal formation of Candida albicans require the Ste20p-like protein kinase CaCla4p. Current biology : CB 7, 539-546 (1997).

27 Santos, M. \& de Larrinoa, I. F. Functional characterization of the Candida albicans CRZ1 gene encoding a calcineurin-regulated transcription factor. Curr Genet 48, 88-100, doi:10.1007/s00294-005-0003-8 (2005).

28 Karababa, M. et al. CRZ1, a target of the calcineurin pathway in Candida albicans. Molecular Microbiology 59, 1429-1451, doi:10.1111/j.1365-2958.2005.05037.x (2006).

29 Kelly, M. T. et al. The Candida albicans CaACE2 gene affects morphogenesis, adherence and virulence. Mol Microbio/ 53, 969-983, doi:10.1111/j.13652958.2004.04185.x (2004). 
30 Desai, P. R., van Wijlick, L., Kurtz, D., Juchimiuk, M. \& Ernst, J. F. Hypoxia and Temperature Regulated Morphogenesis in Candida albicans. PLoS Genetics 11, e1005447-1005431, doi: http://doi.org/10.1371/journal.pgen.1005447 (2015).

31 Hall, R. A. et al. The Mnn2 mannosyltransferase family modulates mannoprotein fibril length, immune recognition and virulence of Candida albicans. PLoS Pathog 9, e1003276, doi:10.1371/journal.ppat.1003276 (2013).

32 Bain, J. M. et al. Candida albicans hypha formation and mannan masking of $\beta$ glucan inhibit macrophage phagosome maturation. mBio 5, e01874, doi:10.1128/mBio.01874-14 (2014).

33 Flint, H. J., Scott, K. P., Louis, P. \& Duncan, S. H. The role of the gut microbiota in nutrition and health. Nature reviews. Gastroenterology \& hepatology 9, 577-589, doi:10.1038/nrgastro.2012.156 (2012).

34 Jacobs, I. Blood lactate. Implications for training and sports performance. Sports Med 3, 10-25 (1986).

35 Netea, M. G. et al. Immune sensing of Candida albicans requires cooperative recognition of mannans and glucans by lectin and Toll-like receptors. J Clin Invest 116, 1642-1650, doi:10.1172/JCl27114 (2006).

36 Ferwerda, B. et al. Human dectin-1 deficiency and mucocutaneous fungal infections. N Engl J Med 361, 1760-1767, doi:10.1056/NEJMoa0901053 (2009).

37 Iliev, I. D. et al. Interactions between commensal fungi and the C-type lectin receptor Dectin-1 influence colitis. Science 336, 1314-1317, doi:10.1126/science.1221789 (2012).

38 Wheeler, R. T., Kupiec, M., Magnelli, P., Abeijon, C. \& Fink, G. R. A Saccharomyces cerevisiae mutant with increased virulence. Proc Natl Acad Sci U S A 100, 2766-2770, doi:10.1073/pnas.0437995100 (2003).

39 Chen, Y. L. et al. Convergent Evolution of Calcineurin Pathway Roles in Thermotolerance and Virulence in Candida glabrata. G3 (Bethesda) 2, 675-691, doi:10.1534/g3.112.002279 (2012).

40 Lev, S., Desmarini, D., Chayakulkeeree, M., Sorrell, T. C. \& Djordjevic, J. T. The Crz1/Sp1 transcription factor of Cryptococcus neoformans is activated by calcineurin and regulates cell wall integrity. PLoS One 7, e51403, doi:10.1371/journal.pone.0051403 (2012).

41 Ene, I. V. et al. Carbon source-induced reprogramming of the cell wall proteome and secretome modulates the adherence and drug resistance of the fungal pathogen Candida albicans. Proteomics 12, 3164-3179, doi:10.1002/pmic.201200228 (2012).

42 Gravelat, F. N. et al. Aspergillus galactosaminogalactan mediates adherence to host constituents and conceals hyphal beta-glucan from the immune system. PLoS Pathog 9, e1003575, doi:10.1371/journal.ppat.1003575 (2013).

43 Rappleye, C. A. \& Goldman, W. E. Fungal stealth technology. Trends Immunol 29, 18-24, doi:10.1016/j.it.2007.10.001 (2008).

44 Noble, S. M. \& Johnson, A. D. Strains and strategies for large-scale gene deletion studies of the diploid human fungal pathogen Candida albicans. Eukaryot Cell 4, 298-309, doi:10.1128/EC.4.2.298-309.2005 (2005).

45 Lewis, L. E. et al. Stage specific assessment of Candida albicans phagocytosis by macrophages identifies cell wall composition and morphogenesis as key 
determinants. PLoS Pathog 8, e1002578, doi:10.1371/journal.ppat.1002578 (2012).

46 Netea, M. G. et al. Increased susceptibility of TNF-alpha lymphotoxin-alpha double knockout mice to systemic candidiasis through impaired recruitment of neutrophils and phagocytosis of Candida albicans. J Immunol 163, 1498-1505 (1999).

47 Graham, L. M. et al. Soluble Dectin-1 as a tool to detect beta-glucans. J Immunol Methods 314, 164-169, doi:10.1016/j.jim.2006.05.013 (2006).

48 Shahana, S. et al. New Clox Systems for rapid and efficient gene disruption in Candida albicans. PLoS ONE 9, e100390, doi:10.1371/journal.pone.0100390 (2014).

49 Inglis DO et al. The Candida Genome Database incorporates multiple Candida species: multispecies search and analysis tools with curated gene and protein information for Candida albicans and Candida glabrata. . Nucleic Acids Res. 40, D667-674 (2012).

50 Bindea, G. et al. ClueGO: a Cytoscape plug-in to decipher functionally grouped gene ontology and pathway annotation networks. Bioinformatics 25, 1091-1093, doi:10.1093/bioinformatics/btp101 (2009).

51 Shannon, P. et al. Cytoscape: a software environment for integrated models of biomolecular interaction networks. Genome Res 13, 2498-2504, doi:10.1101/gr.1239303 (2003).

\section{Figure Legends}

Figure 1: Lactate activates $\beta$-glucan masking in Candida an attenuates phagocytic response. (a) Flow cytometry analysis of $\beta$-glucan exposure. Wild-type Candida albicans, C. dubliniensis, C. Iusitaniae, C. parapsilosis, C. glabrata, and Saccharomyces cerevisiae were grown in glucose or lactate and examined for $\beta$-glucan masking. The plots and Median Fluorescence Intensities (MFI) are shown for glucosegrown cells (red; MFI value in top right of each panel) and lactate-grown cells (blue; MFI value, top left). $\beta$-glucan masking was defined as a $>50 \%$ decrease in MFI. Plots are representative of data collected in two independent replicate experiments. (b) $\beta$-glucan exposure for $C$. albicans cells grown in glucose (red) or glucose + lactate (purple). (c) Percentage of glucose- or lactate-grown $C$. albicans cells phagocytosed by murine BMDmacrophages; $n=4$ donor mice; measurements were performed in triplicate per mouse per condition and analysed using the Mann-Whitney $U$ test; errors bars $=95 \% \mathrm{Cl}$. (d) Percentage of neutrophils recruited to the site of injection in BALB/C mice intraperitoneally infected with wild-type $C$. albicans pre-grown on glucose or lactate. For each group, $n=6$ mice; data were analysed using the Mann-Whitney $U$ test; error bars = 
$95 \% \mathrm{Cl}$. (e) TNF- $\alpha$ and (f) MIP1 $\alpha$ responses from human M1-activated monocytederived macrophages to glucose- and lactate-grown cells; $\mathrm{n}=4$ donors were exposed to triplicate independent samples of Candida cells for each condition; the data were analyzed using Friedman test for matched non-parametric data; error bars $=95 \% \mathrm{Cl}$.

Figure 2: Lactate-induced $\beta$-glucan masking is specific, physiologically relevant, and mediated by Gpr1. (a) $\beta$-glucan exposure for wild-type $C$. albicans cells grown in glucose (red), and glucose plus $2 \%$ D-lactate (blue, top panel) or glucose plus $2 \% \mathrm{~L}$ lactate (blue, bottom panel). Median Fluorescence Intensities (MFI) are indicated at the top of each panel, the value for glucose on the right, and for lactate on the left. Plots are representative of three independent replicate experiments. (b) $\beta$-glucan exposure for $C$. albicans cells incubated for $6 \mathrm{~h}$ in fresh (red; MFI right) or spent (blue; MFI left) culture medium from $\mathrm{J} 774.1$ macrophages ( $36 \mathrm{~h}$, top panel) or Lactobacillus reuteri (overnight culture, bottom panel). Plots are representative of two independent replicate experiments. (c) $\beta$-glucan exposure in $C$. albicans cells grown on glucose (red; MFI, top right) or lactate (blue; MFI, top left) for gpr1 $\triangle$ gpa2 $\Delta$ cells (top); GPR1 GPA2 wild-type cells (bottom); gpr1A cells (top); GPR1 wild-type cells (bottom); clinical isolate S20175.016 containing pACT1-GPR1-GFP (top); S20175.016 containing the control pACT1-GFP vector (bottom). Plots are representative of data collected in three independent replicate experiments. (d) Micrographs showing Gpr1-GFP internalization in C. albicans wild-type cells (SC5314) expressing Gpr1-GFP following $1 \mathrm{hr}$ exposure to glucose, lactate, or methionine at the indicated concentration. Micrographs are representative of three replicate experiments: scale bar $=10 \mu \mathrm{m}$. (e) Gpr1-GFP localization after $1 \mathrm{hr}$ of exposure to $100 \mathrm{mM}$ of the indicated ligand. Micrographs are representative of three replicate experiments: scale bar $=10 \mu \mathrm{m}$. (f) Dose response curves showing $\beta$-glucan exposure for $C$. albicans cells grown for 4 hours in buffered YNB plus the indicated ligand at $2 \mathrm{mM}$ (lactate only), $10 \mathrm{mM}, 100 \mathrm{mM}$, or $150 \mathrm{mM}$. Values were normalized against $0 \mathrm{mM}$ for each condition: $\mathrm{n}=3$; error bars $=95 \% \mathrm{Cl}$. $\mathbf{( g , h ) ~ M a n n a n ~ ( C o n A , ~ r e d ) ~ a n d ~} \beta$-glucan (Fc-Dectin1, green) exposure for (g) wild-type and (h) gpr1 $\Delta$ gpa2 $\Delta$ cells. Micrographs are representative of two replicate experiments: scale bar $=5 \mu \mathrm{m}$. 
Figure 3: Lactate-induced $\beta$-glucan masking is dependent on Crz1, but not calcineurin. (a) Cytometric analysis of $\beta$-glucan exposure in C. albicans cells grown on glucose (red) or glucose plus lactate (blue): CRZ1 wild-type; crz1 $1 \Delta$ mutant; crz1 $1 \Delta+$

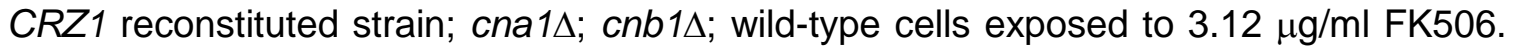
Median Fluorescence Intensities (MFI) are indicated at the top of each panel, the value for glucose on the right, and for lactate or FK506 on the left. Plots are representative of data collected in three independent replicate experiments. (b) Micrographs showing the localization of GFP-Crz1 in wild-type (CAI4+Clp10), cnb1s, and gpr1s gpa2s cells grown in or glucose alone (control), glucose and exposed to $100 \mu \mathrm{M} \mathrm{CaCl}_{2}(1 \mathrm{~h}$ ), or glucose plus $2 \%$ lactate $(4 \mathrm{~h})$. Micrographs are representative of three replicate experiments: scale bar $=5 \mu \mathrm{m}$. (c) Micrographs showing mannan (ConA, red) and $\beta$ glucan (Fc-Dectin1, green) exposure in wild-type and crz1 $\Delta$ cells following growth in glucose or glucose plus lactate. Micrographs are representative of two replicate experiments: scale bar $=5 \mu \mathrm{m}$.

Figure 4: Role of lactate-regulated genes in lactate-dependent $\beta$-glucan masking.

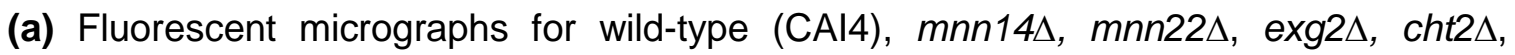
pga26 $\Delta$, and ace $\Delta$ cells stained for chitin (Calcofluor White, blue) and $\beta$-glucan (FcDectin1, green). Micrographs are representative of two replicate experiments: scale bar $=5 \mu \mathrm{m}$. (b) Confocal 3D rendered micrographs showing mannan (ConA, blue) and $\beta$ glucan (Fc-Dectin1, green) exposure in wild-type (SC5314) and ace2A cells. Micrographs are representative of two replicate experiments: scale bar $=5 \mu \mathrm{m}$. (c) $\beta$ -

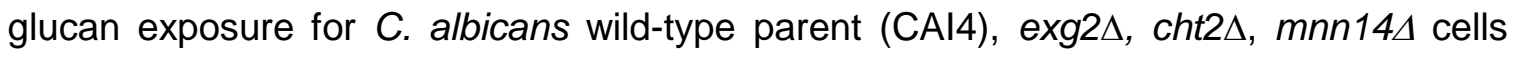
grown on glucose (red) or glucose plus lactate (blue). Median Fluorescence Intensities (MFI) are indicated at the top of each panel, the value for glucose on the right, and for lactate on the left. Data were acquired using a FACSCalibur flow cytometer. Plots are representative of data collected in two independent replicate experiments. (d) $\beta$-glucan exposure for MNN22 parent, mnn224, PGA26 parent and pga26s cells grown on glucose (red) or glucose plus lactate (blue). Median Fluorescence Intensities (MFI) are indicated at the top of each panel, the value for glucose on the right, and for lactate on the left. Data were acquired using a BD Fortessa flow cytometer. Plots are representative of data collected in two independent replicate experiments. 
Figure 5: Regulation of $\beta$-glucan masking in C. albicans. (a) A Crz1 network contributes to lactate-induced gene regulation. Differentially regulated genes are displayed as nodes and classified as either cell wall related (blue) or other (grey), based on their GO annotation. Lactate- and Crz1-regulated clusters are based on the RNA sequencing dataset, and the Ace2 cluster represents genes bound at their promoters by $A c e 2^{30}$. The data used to generate this network in Cytoscape 3.2.1 are presented in Supplementary Table S3. A core set of six genes are regulated by lactate, Crz1 and Ace2, and three of these execute cell wall or cell surface-related functions (MNN22, FET3, HGT10). (b) Model of $\beta$-glucan masking in C. albicans. L-lactate is detected by Gpr1, which in turn signals to Crz1 in a calcineurin independent manner. With the help of Ace2, Crz1 regulates a polygenic response resulting in masking of $\beta$ glucan on the $C$. albicans cell surface. 

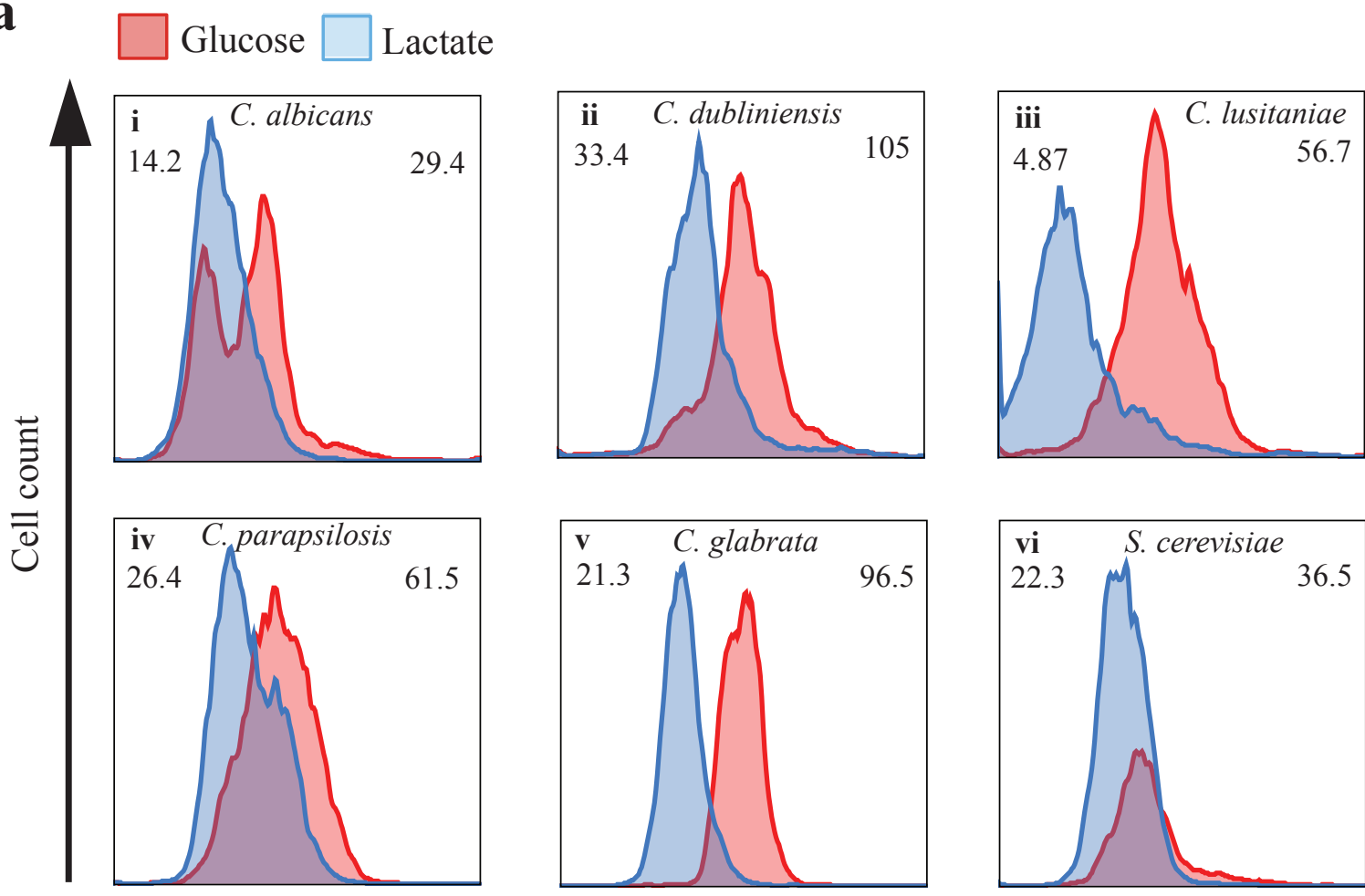

$\beta$-Glucan exposure
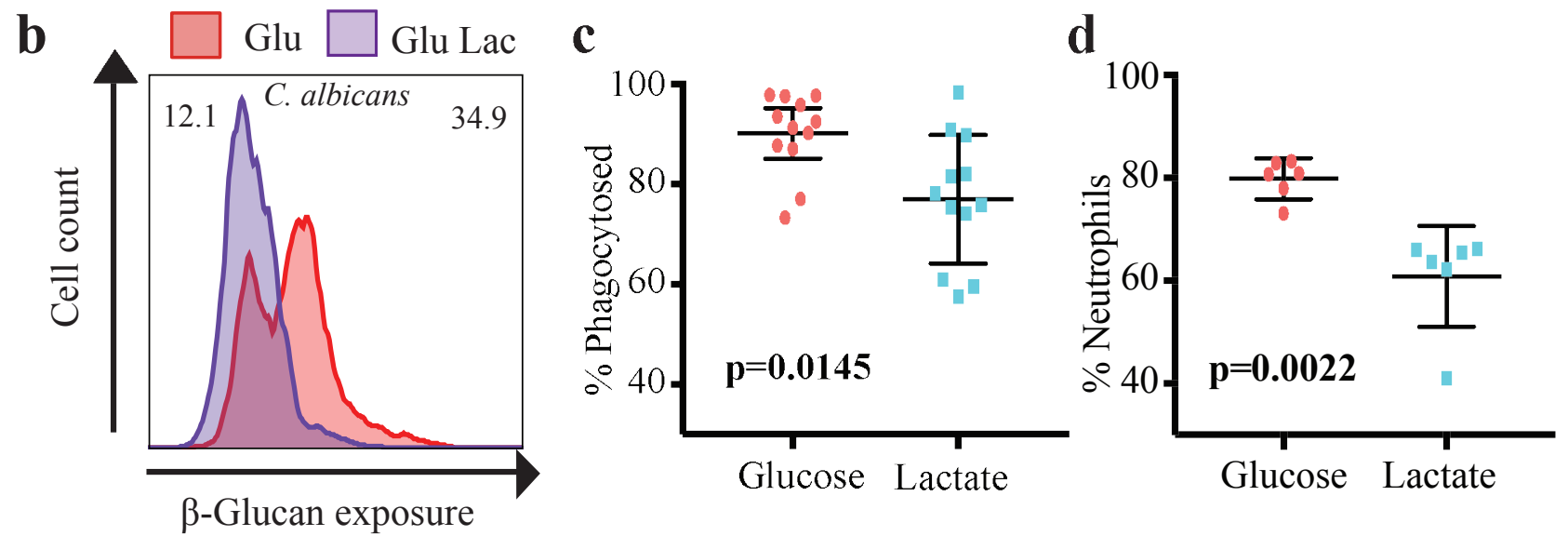

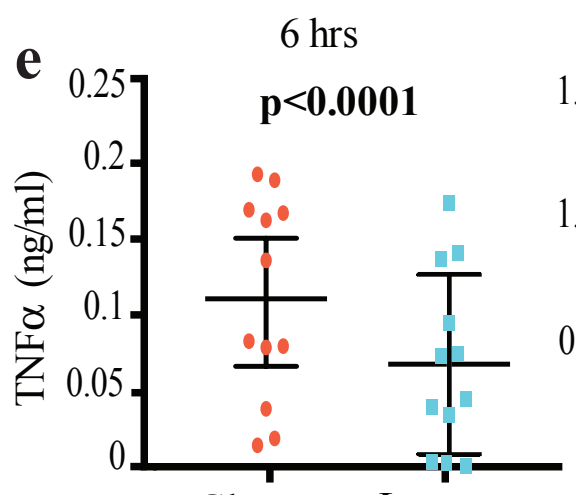

Glucose Lactate
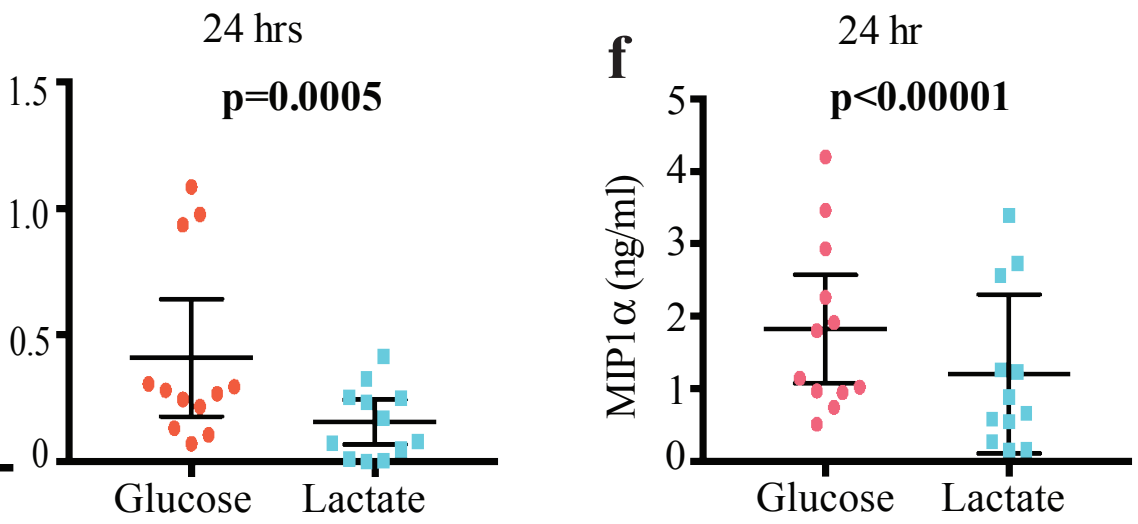
a

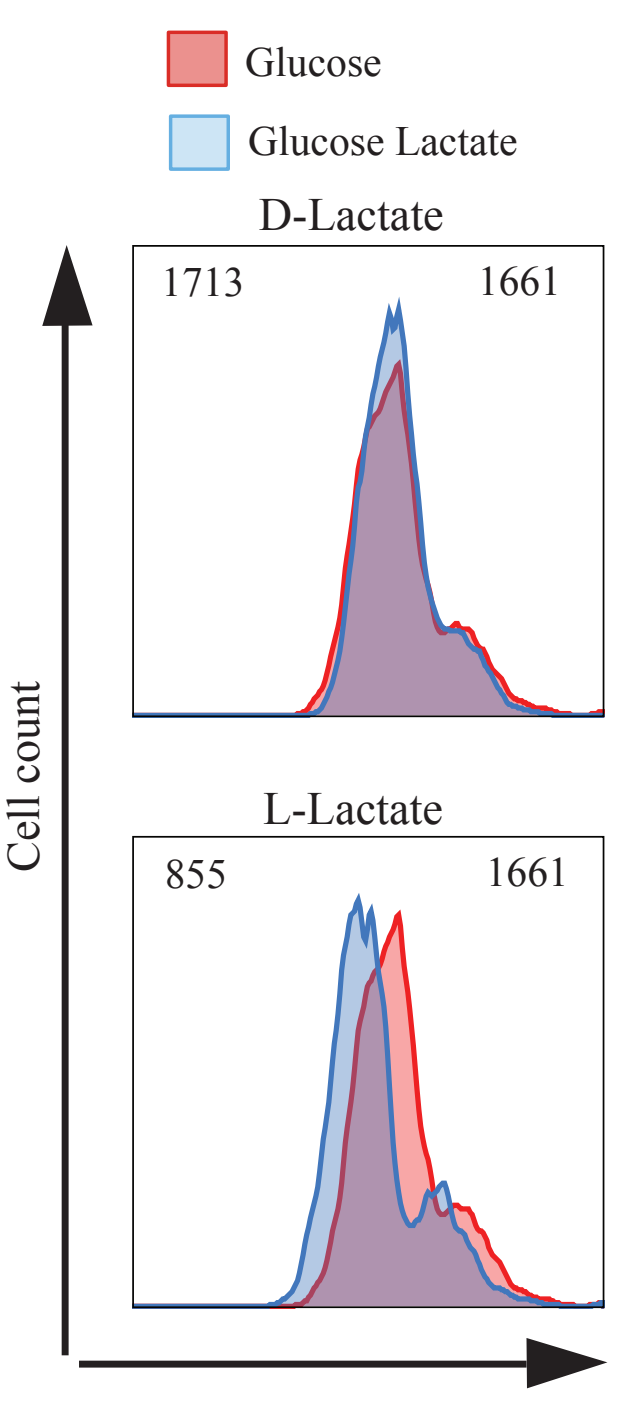

$\beta$-Glucan exposure b

$\square$ Untreated medium $\square$ Treated medium

Macrophage medium

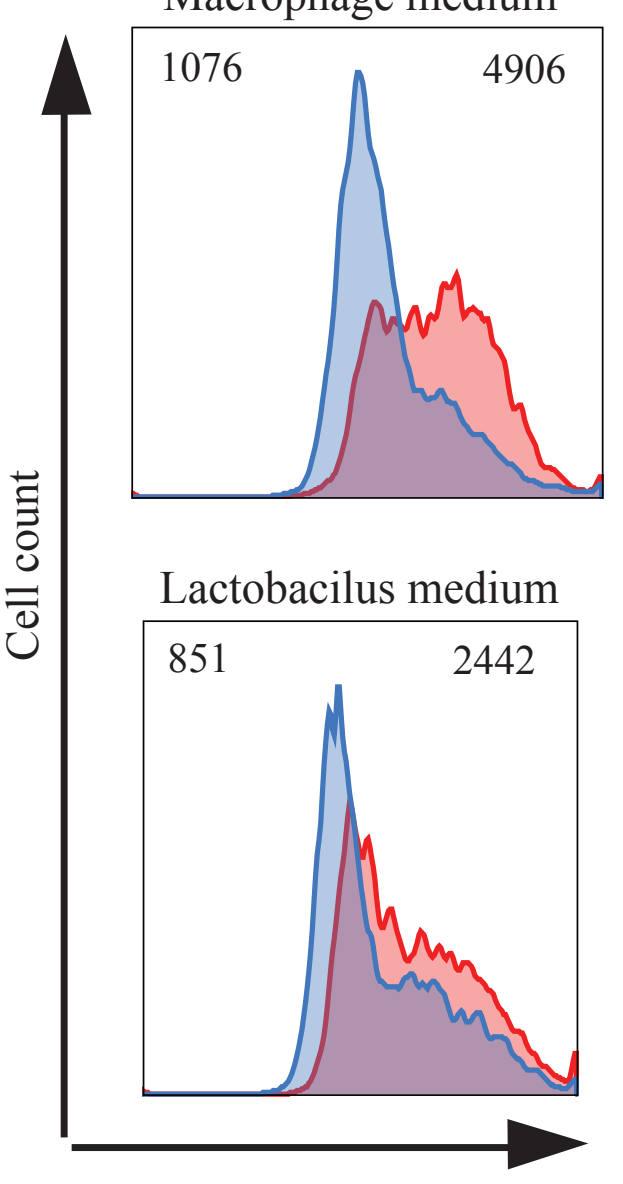

$\beta$-Glucan exposure

c

\section{$\square$ Glucose}

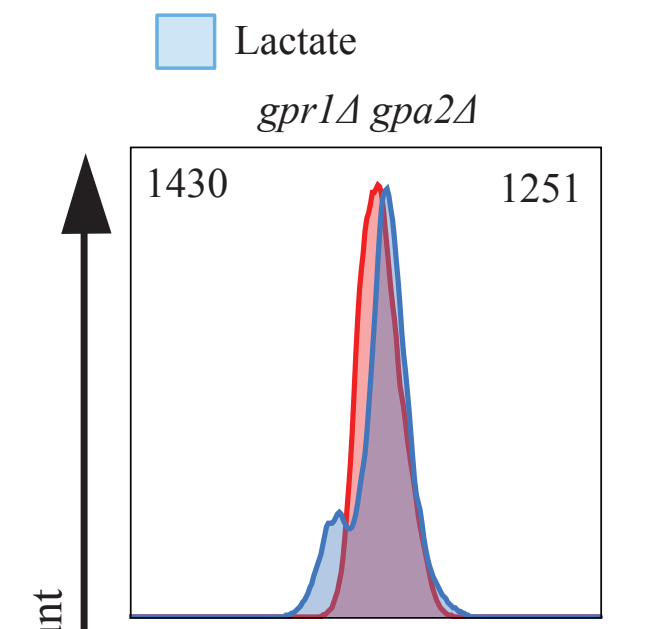

$\overrightarrow{0}$
$\overline{0}$
$\overline{0}$
$\overline{0}$
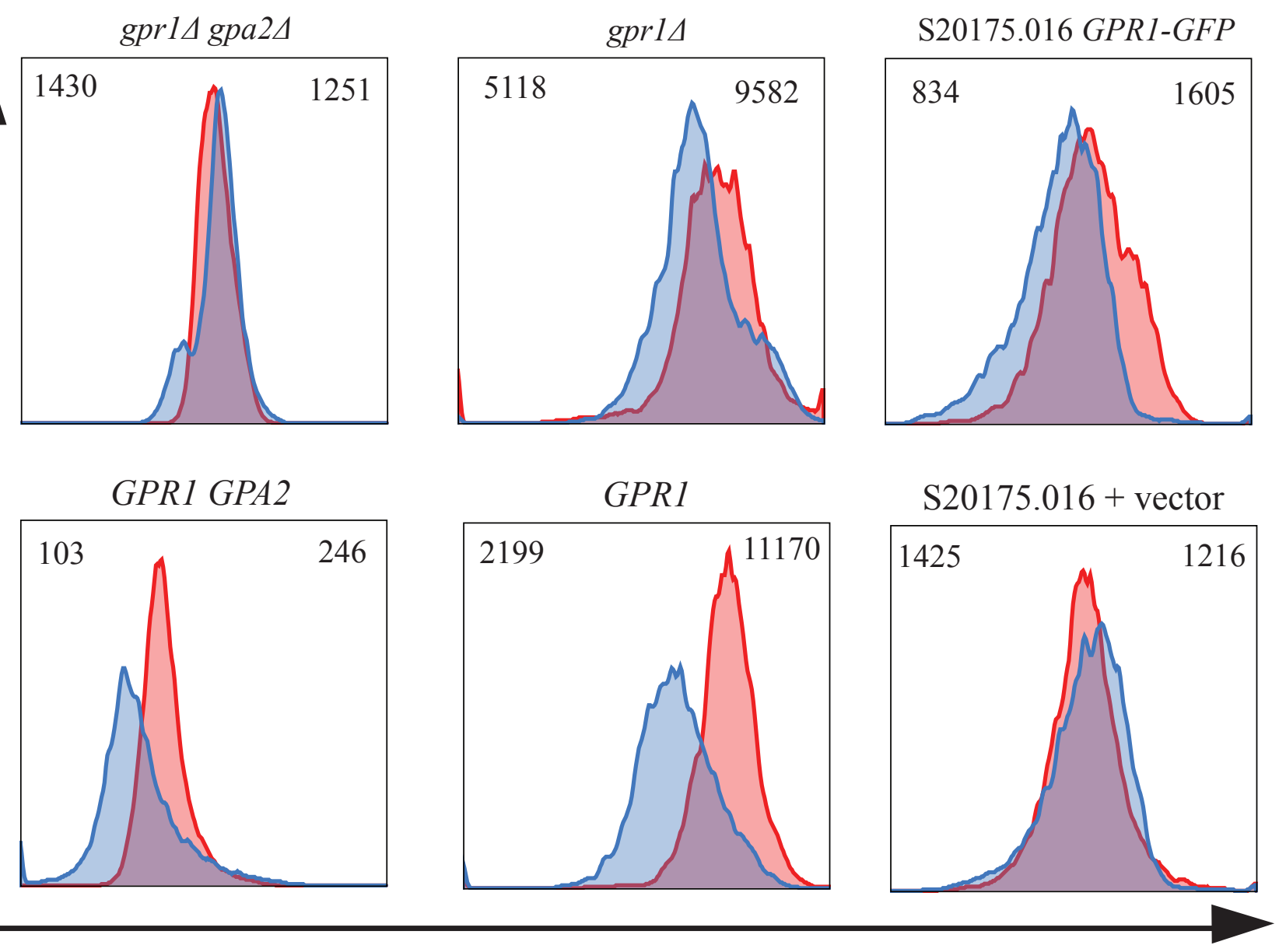

$\beta$-Glucan exposure

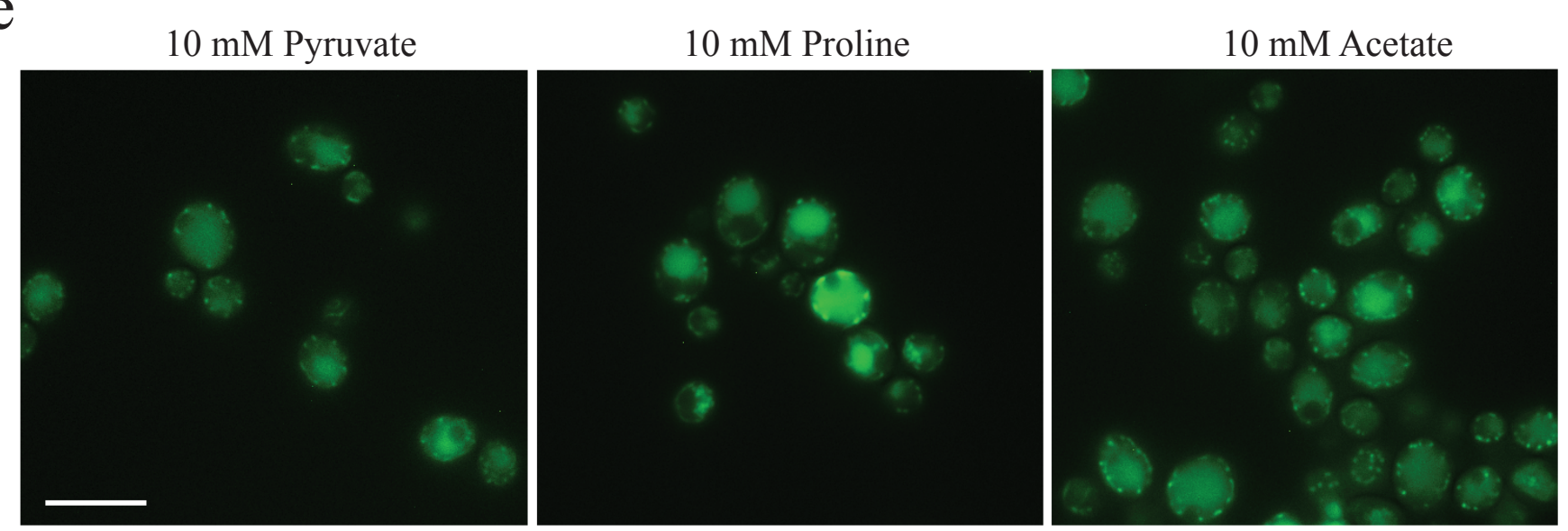

f

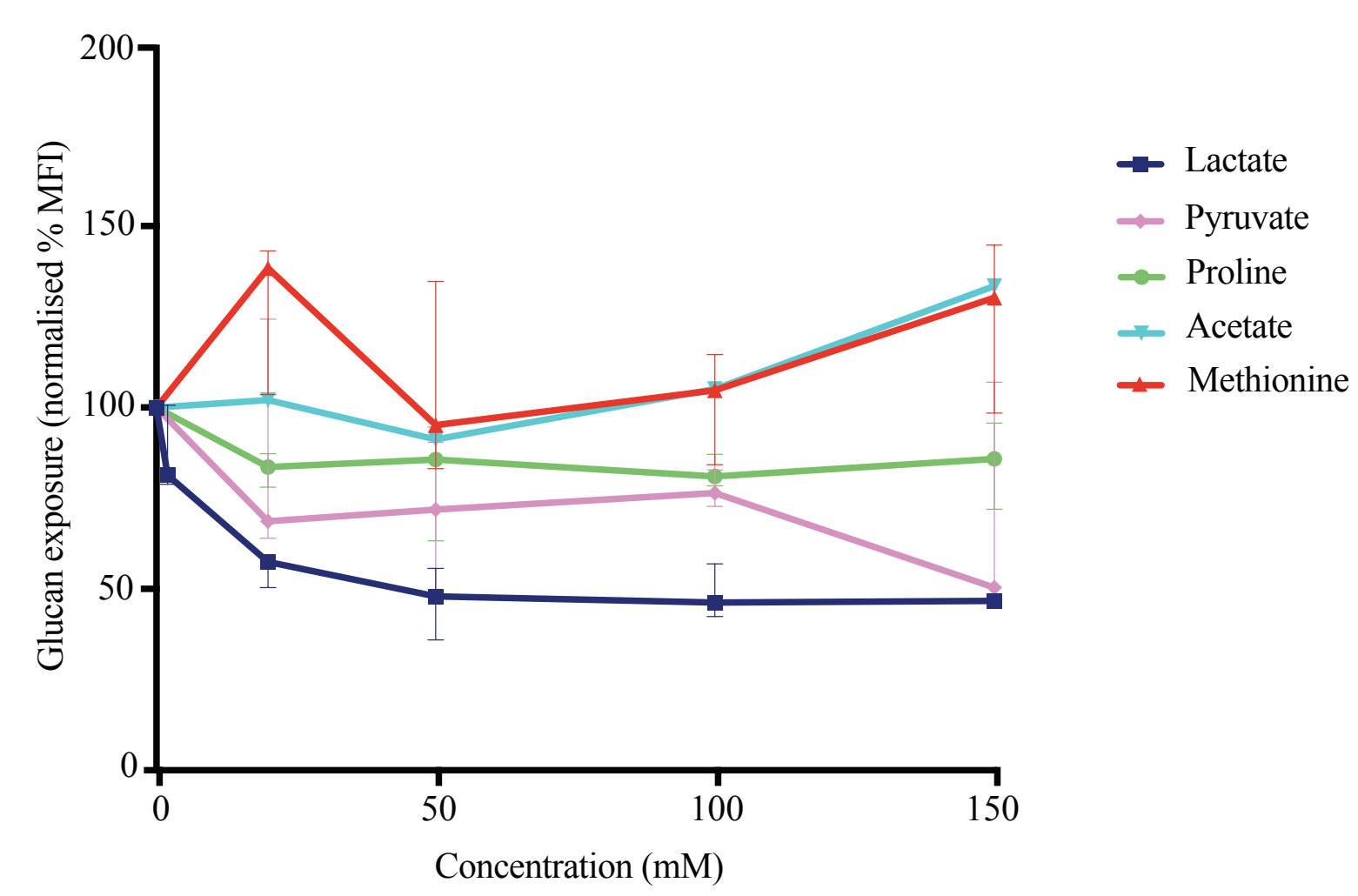

$\mathrm{h}$

e g

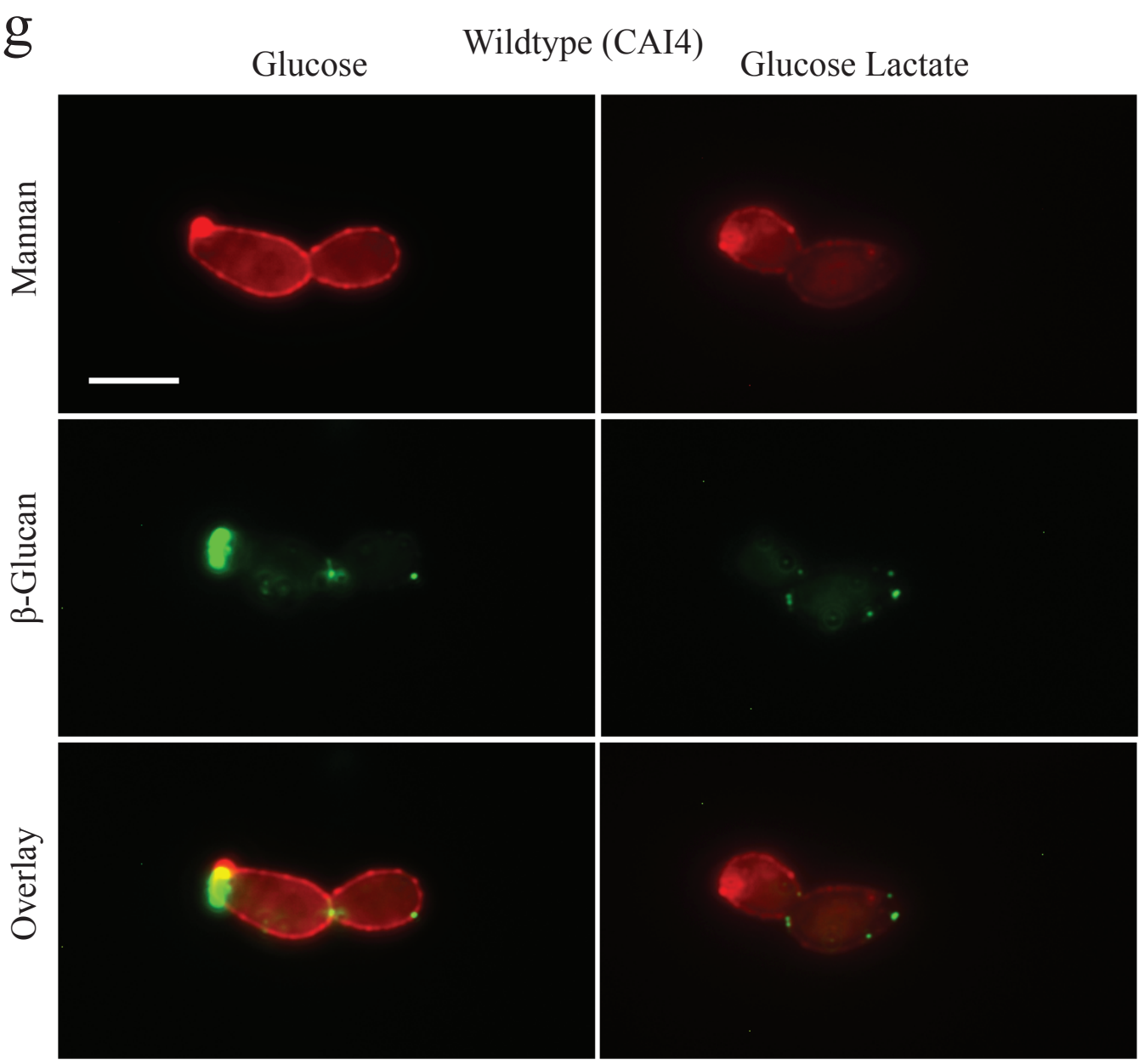

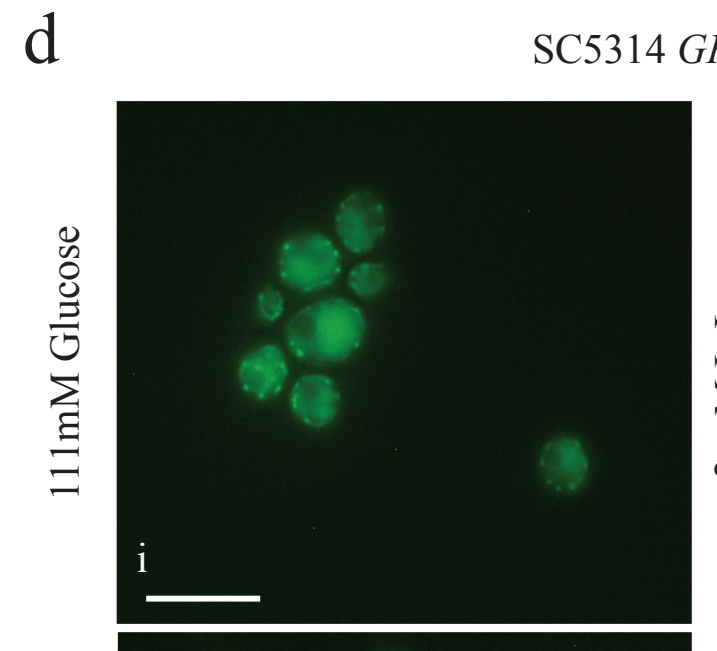
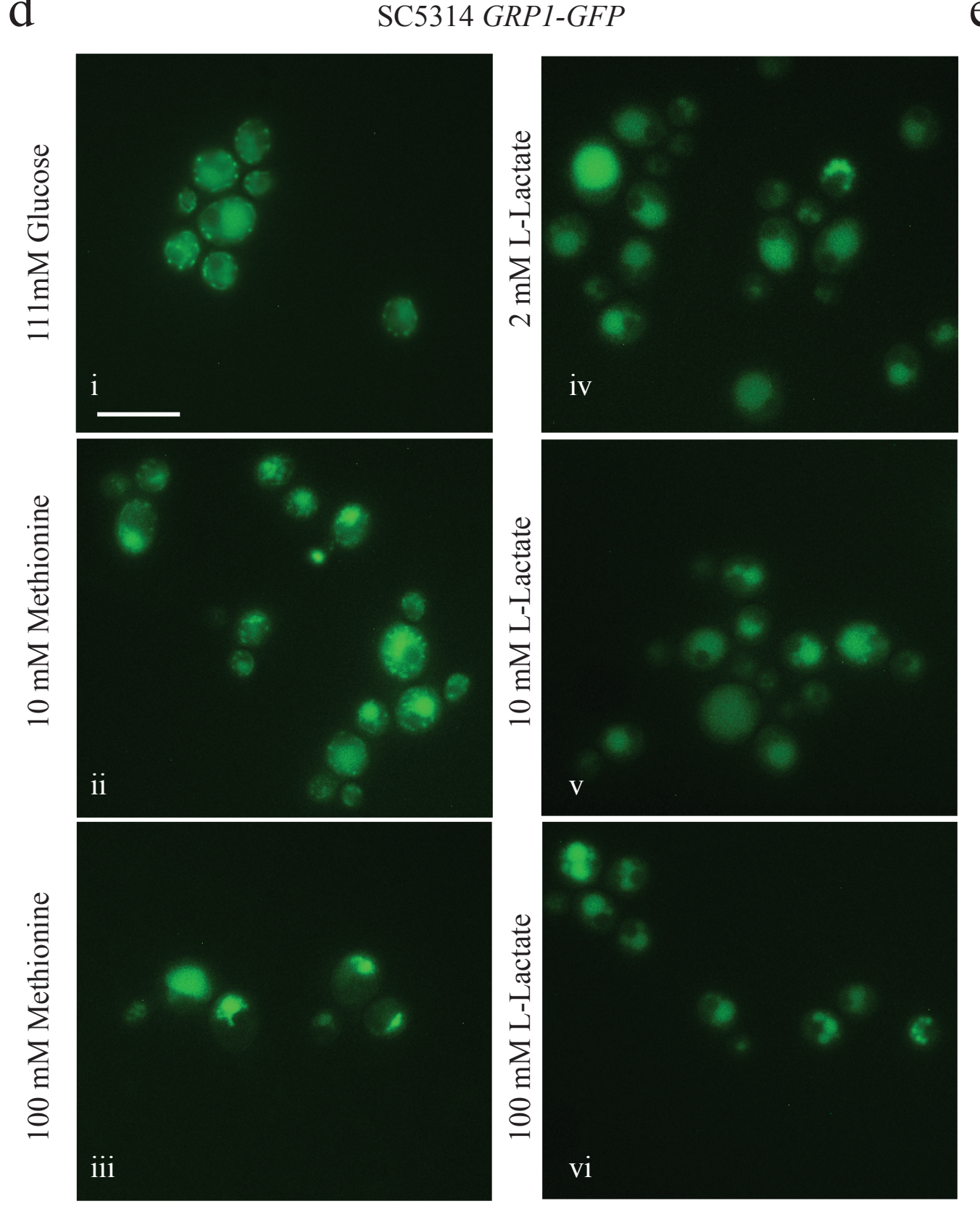

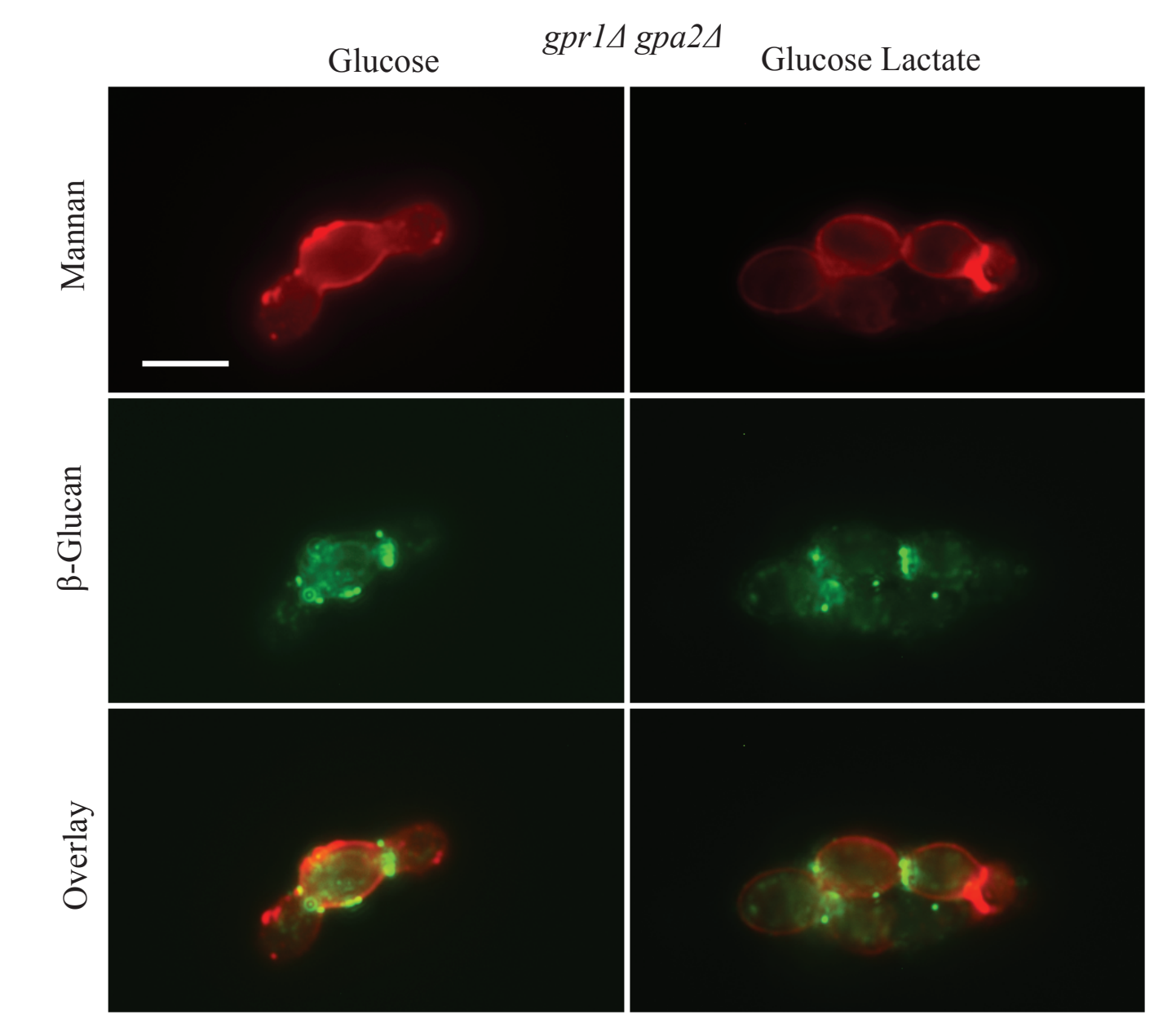


Figure 3

$\mathrm{a}$

$\square$ Glucose $\square$ Glucose Lactate
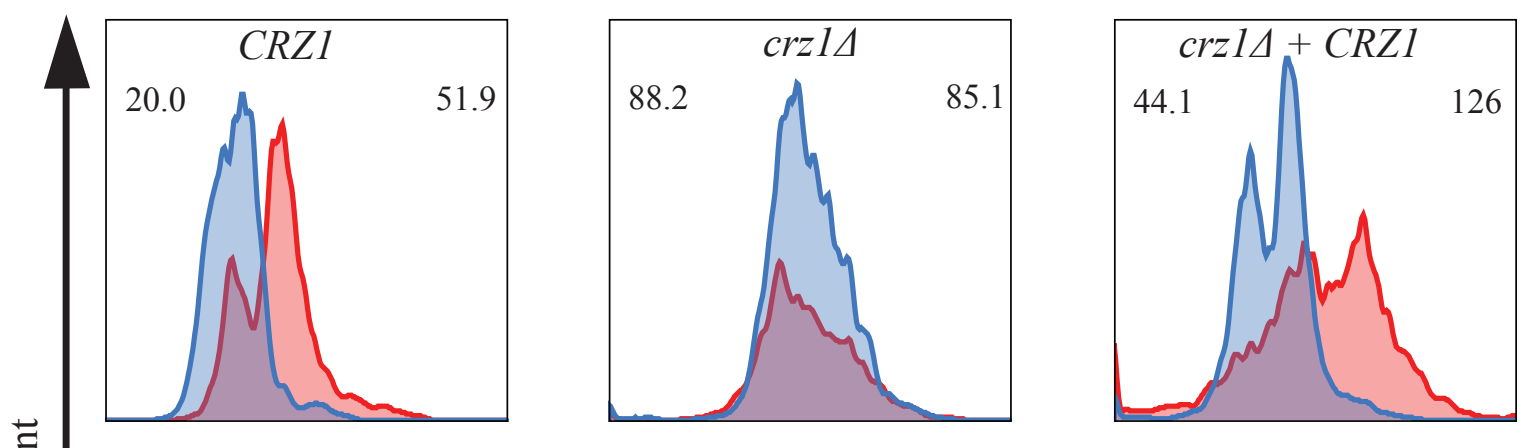

仓

$\square$ Glucose $\square$ Lactate
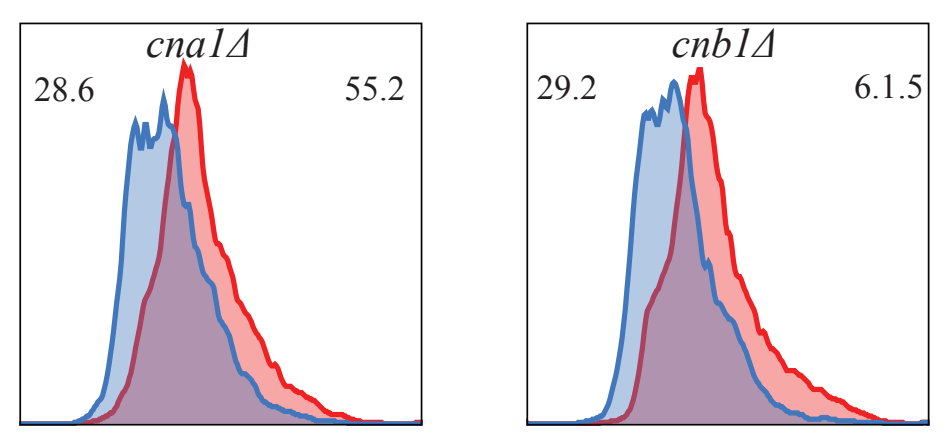

$\square$ Glucose Lactate

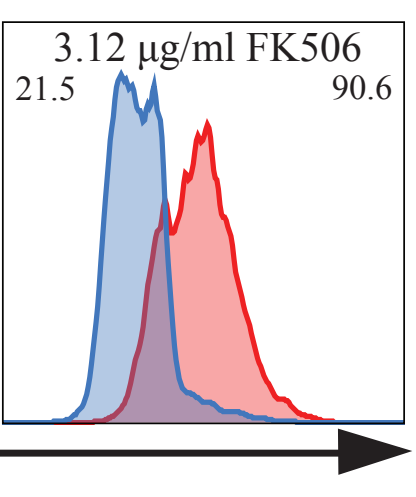

$\beta$-Glucan exposure

b

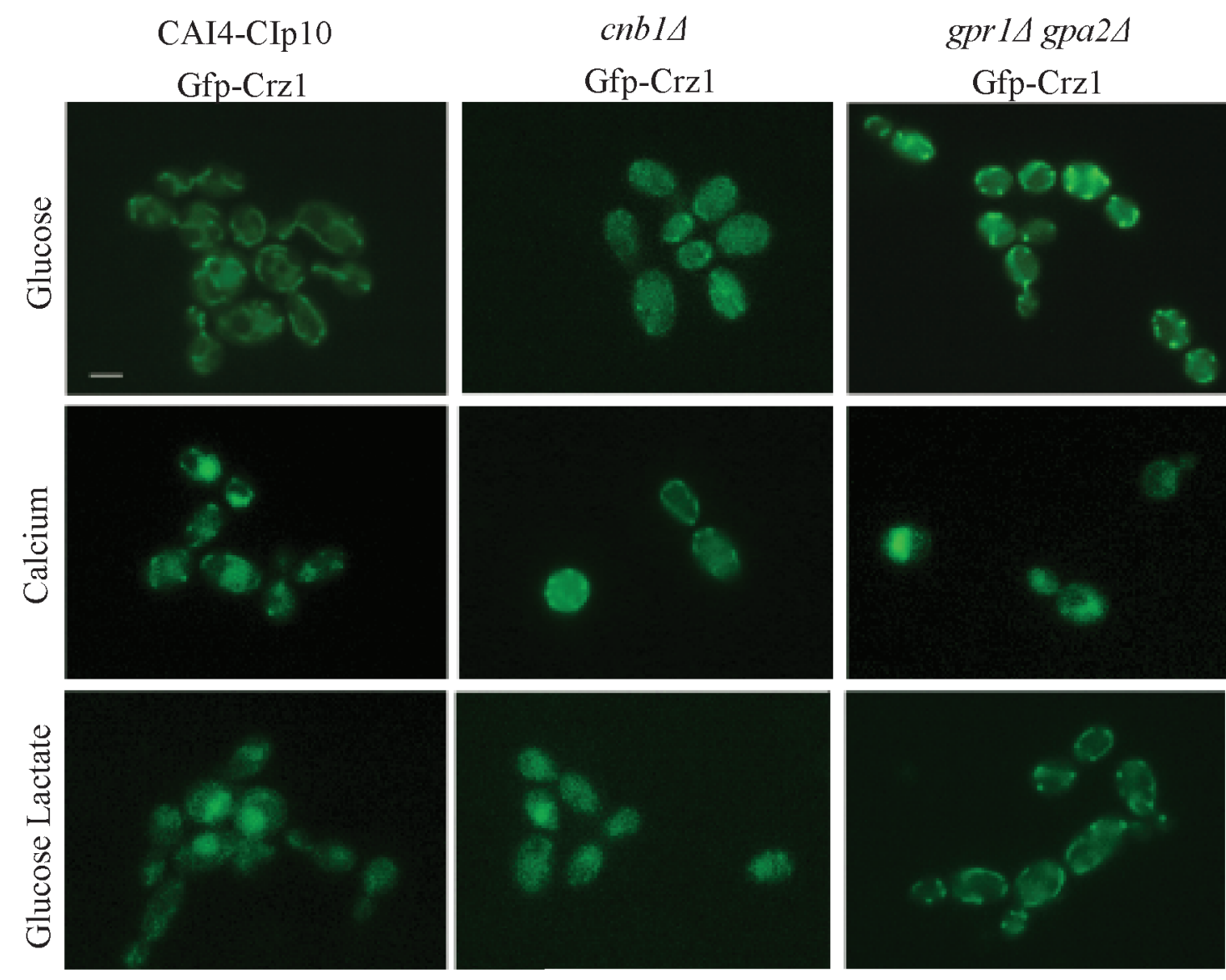

C
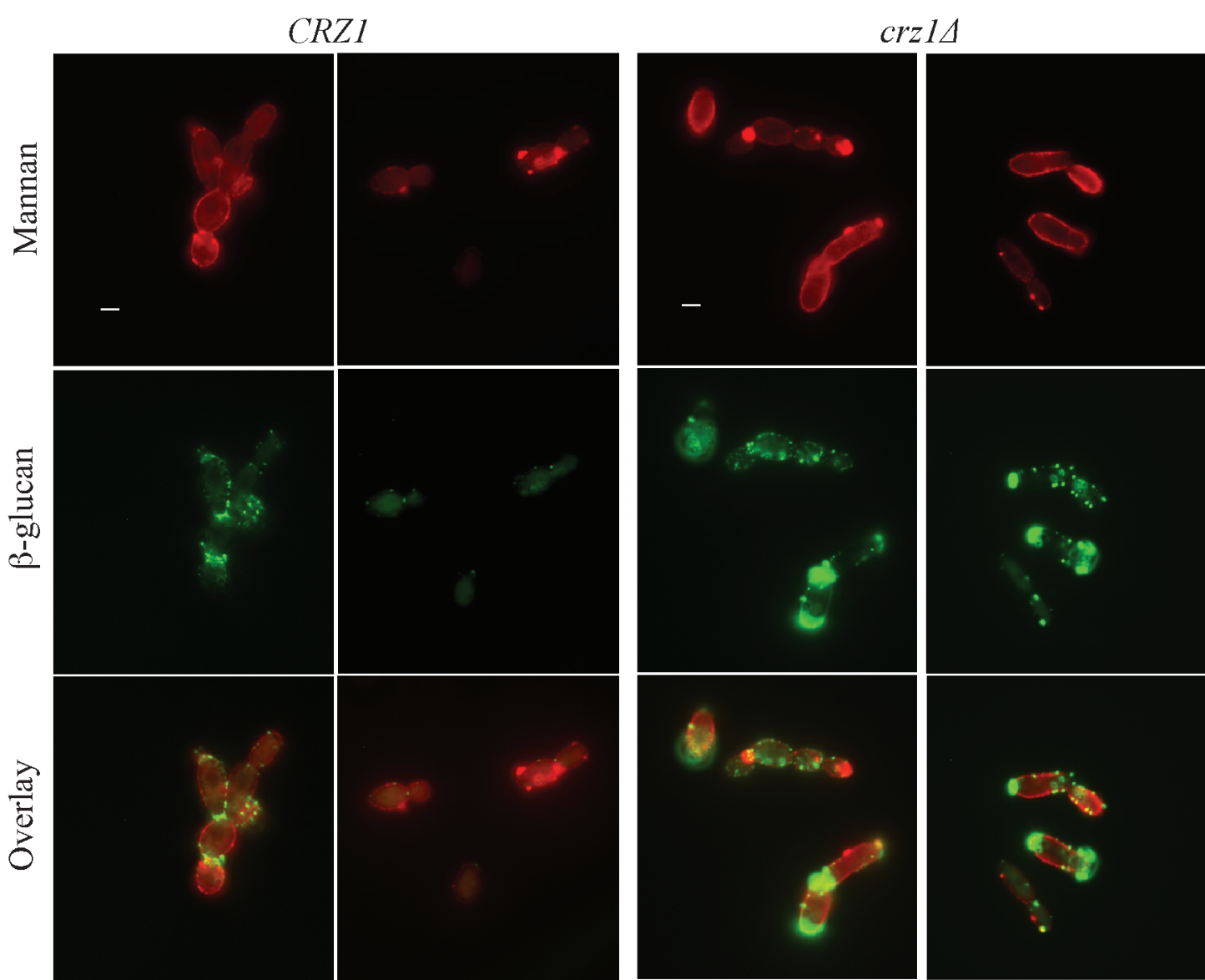

Glucose

Glucose Lactate

Glucose

Glucose Lactate 
Figure 4

a
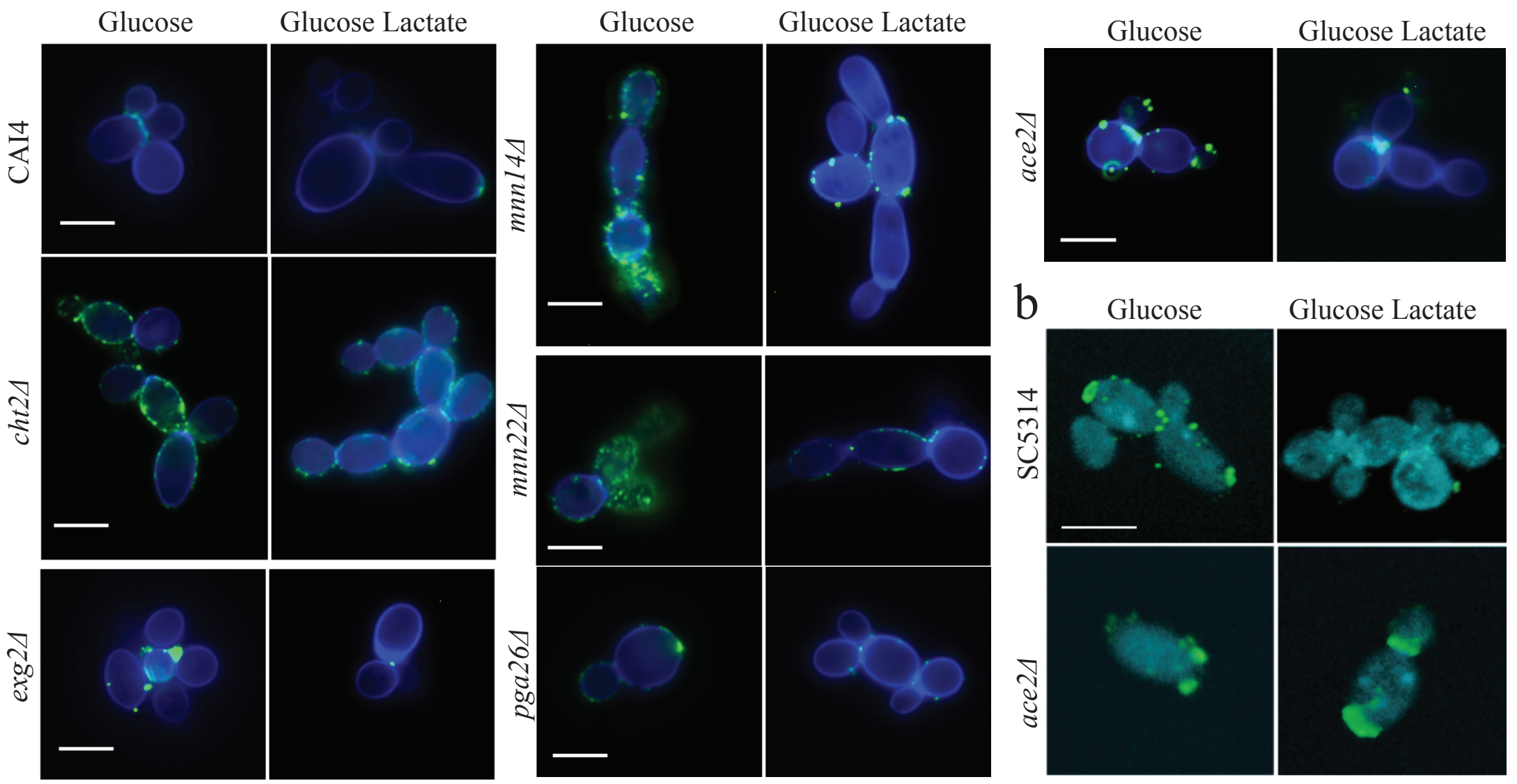

C
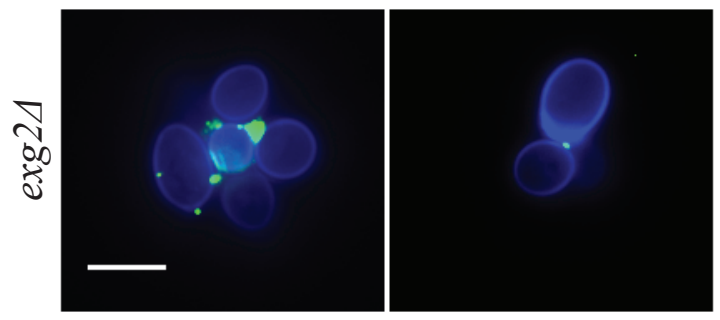

Glucose $\square$ Glucose Lactate
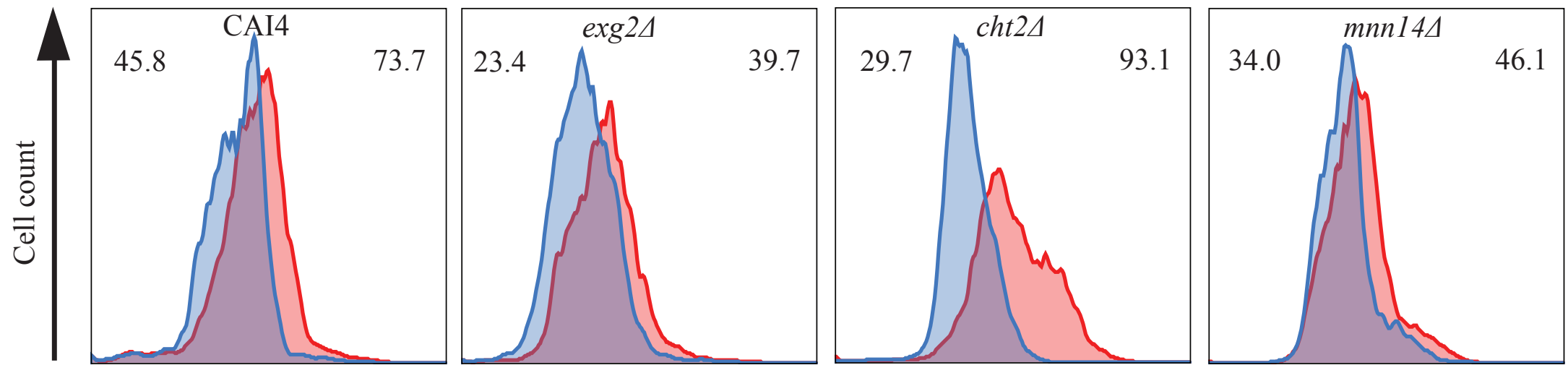

$\beta$-Glucan exposure

d

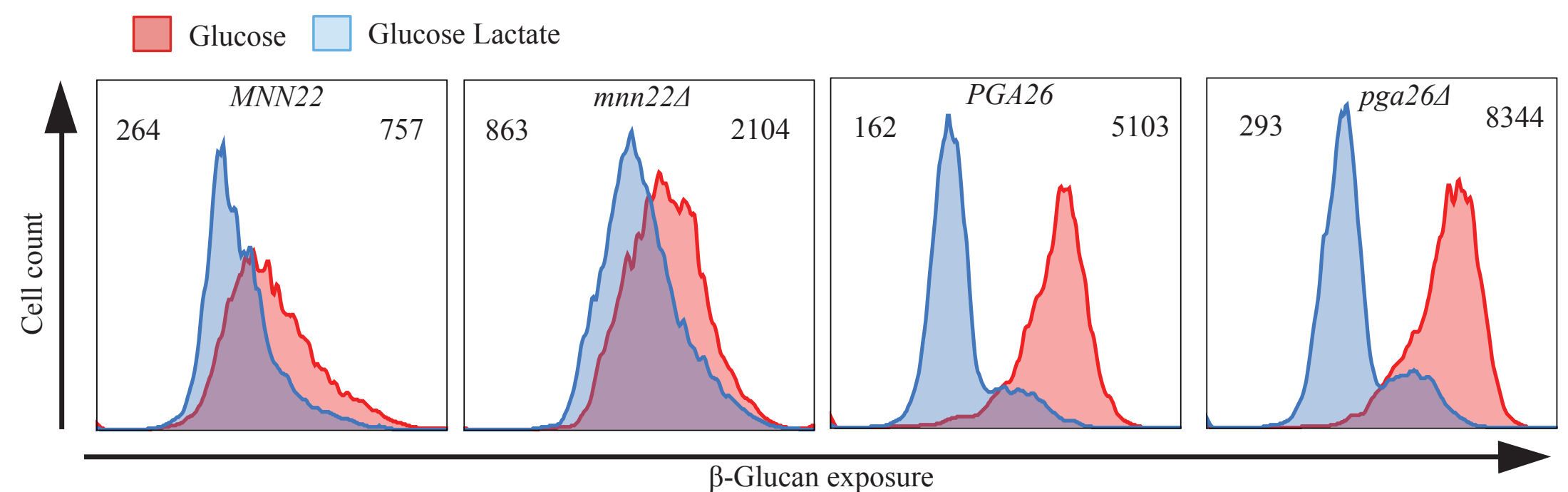


a

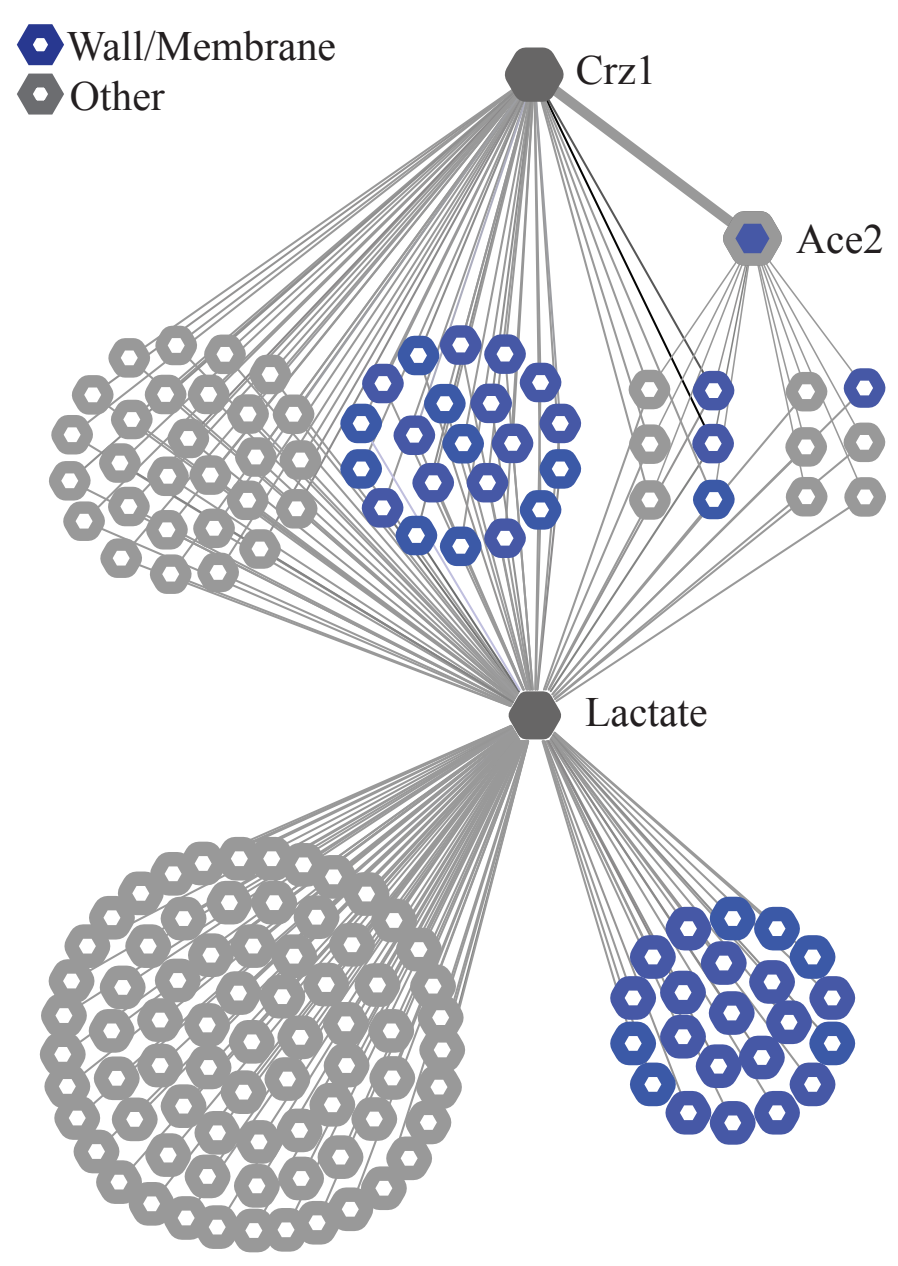

b

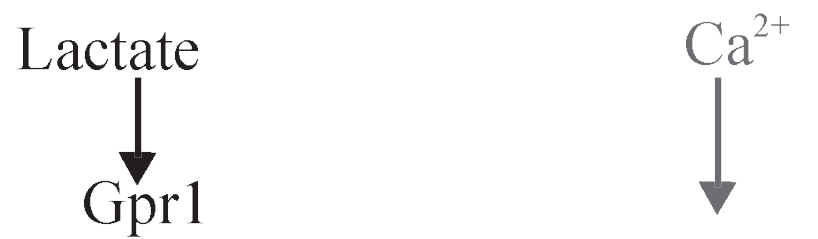

Calcineurin

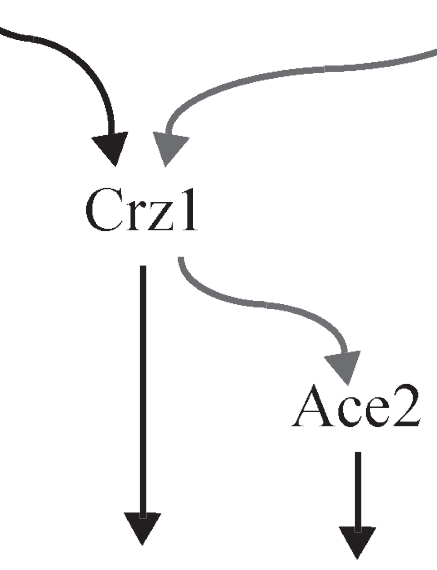

Cell wall remodeling $\downarrow$ $\beta$-glucan masking $\perp$

Immune surveillance 